\title{
The Euro crisis. Causes and symptoms
}

\section{La Euro crisis. Causas y síntomas}

\author{
Christoph S. Weber*
}

\begin{abstract}
The Euro crisis is mainly a consequence of the international financial crisis of 2008. Thereby, the term Euro crisis is misleading as there is no currency crisis. First, the article shows some of the birth defects of the Euro. Second, it shows that the increase in public debt was caused by rescue measures for banks and anti-cyclical fiscal policy. Third, we argue that the Euro crisis is not just one crisis (a sovereign debt crisis) but it is a combination of several macroeconomic crises including a growth crisis, a labour market crisis, a public debt crisis, and a current account crisis.

Keywords: Euro crisis, financial crisis, sovereign debt crisis, unemployment, balance of payment imbalances.
\end{abstract}

Resumen

La crisis del euro es principalmente una consecuencia de la crisis financiera internacional de 2008. Pero el término "crisis del euro" es engañoso ya que no es una crisis de moneda. En primer lugar, el artículo muestra algunos de los defectos originarios en las políticas que dieron nacimiento al euro. En segundo lugar, muestra que el aumento de la deuda pública resulta de las medidas de rescate para los bancos y de la política fiscal anticíclica. En tercer lugar, se sostiene que la crisis del euro no consiste sólo en una crisis (la crisis de la deuda pública), sino que se trata de una combinación de varias crisis macroeconómicas que incluye una crisis de crecimiento, una crisis del mercado de trabajo, una crisis de la deuda pública y una crisis de la balanza de pagos.

Palabras clave: crisis del euro, crisis financiera internacional, crisis de la deuda pública, crisis del mercado de trabajo, crisis de la balanza de pagos.

Recibido: 22 de noviembre de 2013.

Aprobado: 10 de diciembre de 2014.

* Friedrich-Alexander Universitaet Erlangen-Nuernberg, Institute of Economics. Address: Kochstraße 4 (17), 91054 Erlangen, Germany. E-mail: christoph.weber@ fau.de 


\section{Introduction ${ }^{1}$}

At the very beginning of the subprime crisis (2006), few thought that this had the potential to plunge the world economy into its worst post-war recession. The situation changed dramatically when Lehman Brothers went bankrupt in September 2008. The us, Europe and other regions dealt a body blow since many banks had invested in asset backed securities. The us financial crisis spilled over into Europe and this led to a recession in the Eurozone in 2009. However, no one thought that the subprime crisis would result in the most severe crisis post-war Europe had ever faced. In 2009 the financial crisis in Europe turned into a crisis of its own, the socalled Euro crisis. The Euro crisis is mainly economic and political. It is, however, not a crisis of the currency itself. Thus, the label Euro crisis is misleading.

This article aims to work out the main causes of the Euro crisis. As mentioned above the Euro crisis is to a large extent a result of the financial crisis of 2008. Furthermore, the article shows that there is actually not only one macroeconomic crisis in Europe but several. The Euro debt crisis consists of a Gross Domestic Product (GDP) growth crisis, an unemployment crisis, a sovereign debt crisis, and a balance of payment crisis.

This article is structured as follows: the article starts with an overview of the history of the Euro and theoretical consideration regarding the currency union. The fourth section discusses causes of the Euro crisis. The fifth section shows the different components of the Euro crisis. The last section concludes.

\section{History of the Euro}

The Euro had two main predecessors. The first one was the "currency snake" which was created in 1972/73 by the European Economic Community (EEC) as a new system of exchange rates. Within the currency snake

${ }^{1}$ This article is based on a lecture that I gave as a visiting lecturer at the UABC. I would like to thank Martín Arturo Ramírez Urquidy and Ana Bárbara Mungaray Moctezuma for their help. Furthermore, I wish to thank Stuart Jenks, Jürgen Kähler, and Saul Oziel López for very useful comments and suggestions. 
currencies were pegged within a range of $\pm 2.25 \%$. However, this system broke down. The second predecessor was the European Monetary System (EMS) which was founded in 1979 introducing an artificial currency, the so called European Currency Unit. The Ems consisted of 12 members but came under speculative attacks. In consequence, the UK and Italy left the Ems in 1992. At the same time Jacques Delors published his idea of a common currency in Europe. Finally, this plan was established by the Maastricht Treaty 1991/1992 which changed the European Community (EC) substantially. The treaty had three pillars. ${ }^{2}$ Most importantly, the treaty included the EC, which in turn consisted of four parts including the Economic and Monetary Union (EMU). This EMU was established in three stages. The first stage was the European Exchange Rate Mechanism (1990-1994). In the second stage (1994-1999), national central banks were granted (instrument) independence and the European Monetary Institute (EMI) was founded. The EmI was the predecessor of the European Central Bank (ЕСB), which started its work in 1999. The ЕСв is part of the European System of the Central Banks (ЕSCB) which is now in charge of monetary policy in the Eurozone. The Euro was introduced at the very beginning of 1999 as electronic currency only, while people used their domestic currencies from 1999 to 2002. From 2002 onwards the Euro is the sole legal tender in the Eurozone. Any country that wants to become member of the currency area is required to meet the Maastricht criteria. These are: first, inflation rates should not be more than 1.5 percentage points higher than the average of the top three countries. Second, exchange rates have to remain within the EMs bands for two years. Third, average nominal long-term interest rates should not surmount that of the three best performing countries by more than two percentage points. Fourth and most importantly, budget deficits must not be higher than $3 \%$ with respect to GDP and the debt-to-GDP ratio must not be higher than $60 \%$. While the first three criteria were no hurdles for most countries, several countries struggled to meet the fourth criterion. However, budget deficits can relatively easily be reduced by privatizations and other measures. Thus, the focus was mainly on gross public debt. Eventually, most Ec countries managed to meet this requirement. The exceptions were Italy, Belgium, and Greece.

${ }^{2}$ The three pillars are: the European Community, common foreign and security policy, and police and judicial cooperation in criminal matters. 
While Greece was not allowed to introduce the Euro in 1999, Italy and Belgium were, although they did not pass this test. Two years later Greece was allowed to join, despite the fact that Greece's debt-to-GDP was still well above $60 \%$ and (as became known in 2004) its statistics were faked. This spotlights one of the root causes of the Euro crisis. The legal framework, which the founders of the Euro thought would force member countries to maintain fiscal rectitude turned out not to be "written in stone". In fact, politicians can move the goalposts at will (and have done so). During the past 14 years several countries failed to fulfil the public deficit criterion, some repeatedly. However, the Maastricht deficit procedure, which mandates fines for sinner countries, was not always implemented. In 2002 and 2003 France and Germany ran deficits which were too high, but strongarmed their partners in the Euro zone into forgoing punishment. This fatally weakened the legal framework of the Maastricht Treaty. Thus, the Maastricht criteria are merely recommendations, not requirements. The Treaty also included a no bail-out clause meaning that member countries shall not be liable for other countries' debt. The idea that founders of the Euro had —namely that the criteria would be a benchmark that all members would endeavour to meet and that the no bail-out clause prevents the assumption of debt - turned out to be blue-sky thinking.

\section{Optimum currency areas and the Euro zone}

The common currency was mainly a political project, not an economic one. This fact explains the acceptance of countries whose public debt was too high as well as the establishment of the currency area as a whole. There is a broad literature that deals with the question whether countries should form a currency area. According to the impossible trinity model, each country has to select from capital mobility, autonomous monetary policy, and fixed exchange rates. One can only have two of the three. In the Euro zone countries have fixed exchange rates against the other member states and there is full capital mobility. Thus, Emu countries cannot conduct monetary policy autonomously. But why should a country voluntarily give up the right to an autonomous monetary policy? Theory might provide an answer. Robert Mundell (1961) was the first to talk about optimum currency 
areas. The question is always: How can economies deal with asymmetric shocks? Countries with flexible exchange rates can revalue their currencies. Such devaluations make the economy externally more competitive. In a currency area this mechanism is not available. Only the currency as a whole can devalue against other currencies. However, this mechanism can be offset by capital and labour mobility. If one member country is hurt by a negative demand shock (e.g., Spain) and another is confronted with a positive demand shock (e.g., Germany), yields and wages decrease in Spain and increase in Germany. These imbalances can be eliminated if workers and capital move from Spain to Germany. This would eventually lead to increasing prices and wages in Spain and decreasing prices and wages in Germany. However, this depends on the condition that workers and capital freely move and that investors and employees are willing to do so. In theory, the first condition is satisfied in the EMU, which mandates free capital and labour mobility. Capital is in fact mobile, but this does not apply to labour for several reasons.

First and foremost, moving to another country is expensive in comparison to transferring capital between financial centres. However, the biggest obstacle is language. In the 18 Euro countries there are 16 different languages. English is not the working language everywhere and particularly not in the manufacturing sector. Moreover, professional qualifications are not easy to transfer from country to country. While nurses have to study at university in Italy, they are only required to complete a course of training in Germany. And most importantly, most people are simply not willing to move from one country to another as they would have to leave their families and friends behind. However, according to McKinnon (1963), a currency area might still be useful for countries, since the most important factor is openness, not factor mobility. The more open an economy is, the less prices are determined by domestic supply and demand. For a very open economy, revaluation might lead to a worse situation as import prices increase and lead to imported inflation. If import products are an important input for exports, export prices might also have to go up. Moreover, domestic wages might increase as employees ask for a wage mark-up due to higher domestic inflation. Both scenarios would reduce price competiveness. Thus, whether a currency area makes sense depends on the interconnectedness 
of domestic economies. The following table presents some evidence about the role of intra-regional trade of selected regions.

Tables 1 and 2 show average percentage intra-trade of selected regions. First, the table shows that percentage intra-trade within the Eurozone is not that large even though there is no currency risk. In the Free Trade Area of the Americas (FTAA) the proportion of intra-region exports is higher than in the Eurozone, despite the currency risk. Moreover, the main driving force seems to be the European Union (EU) and not the Euro area. The values for the Eu 27 are well above those for the Eurozone even though the Euro zone is smaller. Lastly, one can see that average percentage intra-trade in the Euro area has decreased since 2003. However, the McKinnon criterion of openness is met for the largest part.

A third theory dealing with currency areas comes from Kenen (1969). According to him, asymmetric shocks are not that problematic if countries are highly specialized in producing several goods. A more diversified economy has also a more diversified export sector. This means that an asymmetric shock affects only part of the economy. Eventually, shocks should balance themselves out. This means that fixed exchange rates are useful for countries which produce a variety of goods. There are only some studies that discuss whether this applies for Euro countries. According to Baldwin and Wyplosz (2004), product diversification is reasonably high in Europe. However, there are large differences between countries.

Taking into account these theoretical assumptions, one could doubt that the Euro area is an optimum currency area. However, other currency areas like the us are also sub-optimal with respect to the criteria named above. On the other hand, in the us there exists a system of money transfers from prosperous regions to less productive ones. This is not the case in the Euro area as every country conducts its own fiscal and economic policy. But why did countries like Greece want to become members of the currency area even if they were aware of possible problems? The main reason is possibly that these countries hoped that interest rates for domestic debtors (including the government) would decline since, once they were in the Eurozone. ${ }^{3}$ This is exactly what happened after the introduction

${ }^{3}$ There are, of course, other benefits of currency area. These include, for instance, lower transaction costs and higher price stability. For a discussion of benefits and costs of a monetary union in Europe see De Grauwe (2012). 


\begin{tabular}{|c|c|c|c|c|}
\hline$\underset{\curvearrowright}{\approx}$ & $\begin{array}{l}\infty \\
\dot{v} \\
0\end{array}$ & $\begin{array}{l}0 \\
\dot{q}\end{array}$ & 78 & $\stackrel{+}{+}$ \\
\hline$\underset{\approx}{\approx}$ & $\begin{array}{l}n \\
\dot{0}\end{array}$ & $\stackrel{+}{\stackrel{4}{7}}$ & $\begin{array}{l}0 \\
10 \\
10\end{array}$ & 20 \\
\hline$\stackrel{\overbrace{}}{\stackrel{\overbrace{}}{\sigma}}$ & $\begin{array}{l}\infty \\
+ \\
\end{array}$ & $\stackrel{\wedge}{\infty}$ & $\begin{array}{l}\infty \\
\dot{+} \\
10\end{array}$ & ?ִ \\
\hline$\stackrel{\overbrace{}}{\delta}$ & $\begin{array}{l}n \\
\ddot{0}\end{array}$ & $\overrightarrow{0}$ & $\begin{array}{c}\text { Y } \\
\stackrel{10}{10}\end{array}$ & $\stackrel{+}{0}$ \\
\hline 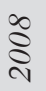 & $\begin{array}{l}0 \\
0 \\
0\end{array}$ & $\begin{array}{l}0 \\
0 \\
10\end{array}$ & $\begin{array}{l}n \\
\ddot{0} \\
20\end{array}$ & $\stackrel{\theta}{\forall}$ \\
\hline 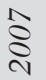 & 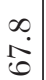 & $\begin{array}{l}0 \\
0 \\
0\end{array}$ & $\begin{array}{l}\infty \\
0 \\
0\end{array}$ & ج8 \\
\hline 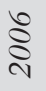 & 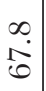 & $\overbrace{10}^{\infty}$ & $\overrightarrow{8}$ & $\begin{array}{c}0 \\
20\end{array}$ \\
\hline$\stackrel{n}{\stackrel{n}{2}}$ & \begin{tabular}{l}
0 \\
\multirow{1}{0}{}
\end{tabular} & $\frac{20}{20}$ & $\begin{array}{l}10 \\
8 \\
8\end{array}$ & $\stackrel{\theta}{\theta}$ \\
\hline$\underset{\sim}{\stackrel{D}{~}}$ & $\begin{array}{l}0 \\
\infty \\
0 \\
0\end{array}$ & $\frac{\infty}{\infty}$ & \begin{tabular}{l}
$\dddot{y}$ \\
\hdashline \\
8
\end{tabular} & $\begin{array}{l}9 \\
20\end{array}$ \\
\hline$\underset{\sim}{\stackrel{m}{\vartheta}}$ & $\vec{\infty}$ & $\underset{10}{\infty}$ & \begin{tabular}{l}
$\mathscr{y}$ \\
\hdashline \\
8
\end{tabular} & $\begin{array}{l}20 \\
20\end{array}$ \\
\hline$\underset{\curvearrowright}{\stackrel{\Upsilon}{\curvearrowright}}$ & Ð & $\begin{array}{l}\text { Oִ } \\
0 \\
10\end{array}$ & $\stackrel{0}{0}$ & $\ddot{0}$ \\
\hline$\underset{\sim}{\curvearrowright}$ & $\frac{1}{6}$ & $\ddot{0}$ & $\ddot{8}$ & $\begin{array}{l}+1 \\
20\end{array}$ \\
\hline 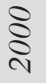 & $\begin{array}{l}10 \\
10 \\
0\end{array}$ & ب0. & $\frac{n}{0}$ & $\stackrel{\infty}{+}$ \\
\hline Әे & $\begin{array}{l}\infty \\
\infty \\
0\end{array}$ & $\begin{array}{l}\hat{N} \\
\text { ov } \\
20\end{array}$ & $\stackrel{0}{0}$ & $\begin{array}{l}0 \\
1\end{array}$ \\
\hline $\begin{array}{l}\infty \\
\curvearrowright \\
\approx\end{array}$ & 今. & $\frac{9}{10}$ & $\begin{array}{l}\not 1 \\
\infty \\
\infty\end{array}$ & $\begin{array}{l}\infty \\
\stackrel{N}{N}\end{array}$ \\
\hline$\widehat{\widehat{\Omega}}$ & $\begin{array}{l}0 \\
10 \\
0\end{array}$ & @̊. & $\begin{array}{l}0 \\
\dot{0} \\
20\end{array}$ & $\begin{array}{r}+ \\
0\end{array}$ \\
\hline$\stackrel{\Xi}{\beth}$ & 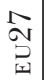 & 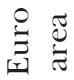 & 苦 & $\begin{array}{l}0 \\
0\end{array}$ \\
\hline
\end{tabular}

\begin{tabular}{|c|c|c|c|c|}
\hline$\frac{v}{\sigma}$ & $\begin{array}{l}70 \\
20 \\
20\end{array}$ & $\stackrel{20}{\mathscr{H}}$ & $\begin{array}{l}\stackrel{H}{*} \\
\stackrel{v}{*}\end{array}$ & $\vec{\sigma}$ \\
\hline$\underset{\approx}{\approx}$ & $\begin{array}{l}\infty \\
10 \\
10\end{array}$ & $\overrightarrow{\ddot{q}}$ & $\stackrel{?}{\not}$ & $\stackrel{?}{?}$ \\
\hline 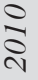 & $\begin{array}{l}10 \\
10 \\
10\end{array}$ & $\stackrel{\leftrightarrow}{\underset{H}{*}}$ & $\stackrel{\not r}{\vec{q}}$ & $\stackrel{\sim}{\infty}$ \\
\hline 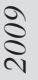 & $\begin{array}{l}n \\
8 \\
8\end{array}$ & $\begin{array}{l}o y \\
\mathscr{f}\end{array}$ & $\stackrel{\text { ๙ }}{\not}$ & $\stackrel{0}{\infty}$ \\
\hline \begin{tabular}{l}
$\infty$ \\
\multirow{2}{2}{}
\end{tabular} & $\begin{array}{l}0 \\
0 \\
20\end{array}$ & $\begin{array}{l}10 \\
18 \\
7\end{array}$ & $\stackrel{F}{\rightleftarrows}$ & $\begin{array}{l}\mathscr{y} \\
\infty\end{array}$ \\
\hline હิ & $\frac{10}{6}$ & 亲 & $\stackrel{\infty}{\underset{\sim}{+}}$ & $\begin{array}{l}\infty \\
\infty\end{array}$ \\
\hline 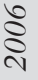 & $\begin{array}{l}0 \\
8 \\
8\end{array}$ & $\begin{array}{l}0 \\
\dot{q}\end{array}$ & $\begin{array}{l}\stackrel{0}{\text { v }} \\
\stackrel{\text { f }}{ }\end{array}$ & $\begin{array}{l}0 \\
\dot{\sigma}\end{array}$ \\
\hline 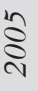 & ?ִ & $\stackrel{\infty}{\stackrel{\infty}{f}}$ & $\begin{array}{l}o \\
\dot{v}\end{array}$ & $\begin{array}{l}\stackrel{0}{\sigma}\end{array}$ \\
\hline$\underset{8}{2}$ & $\begin{array}{c}0 \\
0 \\
0\end{array}$ & $\begin{array}{l}\stackrel{0}{q} \\
\stackrel{+}{+}\end{array}$ & $\begin{array}{l}o \dot{y} \\
\stackrel{y}{f}\end{array}$ & $\begin{array}{l}\infty \\
\infty \\
\infty\end{array}$ \\
\hline$\stackrel{m}{\tilde{\varepsilon}}$ & कृ & ت. & $\stackrel{0}{\infty}$ & $\underset{\infty}{+\infty}$ \\
\hline 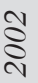 & $\begin{array}{l}\infty \\
0 \\
0\end{array}$ & تே. & $\begin{array}{l}0 \\
10 \\
\stackrel{1}{f}\end{array}$ & $\vec{\sigma}$ \\
\hline $\begin{array}{c}\grave{\delta} \\
\approx\end{array}$ & $\begin{array}{l}\mathcal{v} \\
\text { vi } \\
v\end{array}$ & \begin{tabular}{l}
$\mathscr{Y}$ \\
$\infty$ \\
\multirow{+}{*}{}
\end{tabular} & $\begin{array}{l}0 \\
\dot{0}\end{array}$ & $\begin{array}{l}0 \\
\infty\end{array}$ \\
\hline$\stackrel{\overbrace{}}{\curvearrowright}$ & $\stackrel{1}{0}$ & $\stackrel{\infty}{\stackrel{\infty}{f}}$ & $\ddot{q}$ & $\vec{\sigma}$ \\
\hline ڤे & $\begin{array}{l}0 \\
\dot{+0}\end{array}$ & تי் & $\begin{array}{l}10 \\
\mathscr{0}\end{array}$ & ơ \\
\hline$\stackrel{\infty}{2}$ & $\begin{array}{l}0 \\
\dot{\theta}\end{array}$ & $\begin{array}{l}0 \\
\stackrel{0}{0}\end{array}$ & $\begin{array}{l}0 \\
\dot{0}\end{array}$ & $\underset{\infty}{\infty}$ \\
\hline$\widehat{\widehat{\sigma}}$ & $\begin{array}{c}0 \\
0 \\
0\end{array}$ & $\begin{array}{l}\infty \\
\stackrel{\infty}{+}\end{array}$ & $\begin{array}{l}0 \\
\dot{\varphi}\end{array}$ & $\vec{\sigma}$ \\
\hline$\stackrel{2}{2}$ & 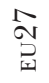 & 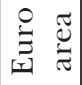 & 这 & نِ \\
\hline
\end{tabular}


of the Euro. To summarize, the project of a common currency was a political idea. It was politicians who decided to construct the Euro and to let countries take part that did not meet all criteria. We should bear in mind that the EMU has had some defects by its very nature. These defects did not cause the crisis, but they did magnify here and there.

\section{Causes of the Euro crisis}

In the last section, we have seen the institutional setting of the Euro zone. It was already mentioned that some countries breached the Maastricht criteria. Thus, the question arises of whether the current crisis is a consequence of excessive government spending. Here, it is instructive to look at government debt and deficits. Tables 3 and 4 show public deficit and debt of all Euro member states.

Bold numbers in tables 3 and 4 indicate breaches of the Maastricht criteria. Some things are noteworthy. First, Greece's and Portugal's public deficit was above the target in every single year of Euro membership. Even countries like Germany failed to meet the deficit criterion several times. On the other hand, countries like Spain and Ireland which later faced grave difficulties were "model students" regarding public deficits until 2008. They even achieved budget surpluses in some years. However, Ireland and Spain could have done much better as tax revenues were high due to credit and housing booms (Lane, 2012). From 2009 onwards most countries failed to meet the $3 \%$ criterion. The data about public debt tells a similar story. Austria, Belgium, Greece, and Italy never met the debt requirement. Germany met the requirement only in one year. Spain and Ireland were again among the best performing countries and could even reduce their debt-to-GDP ratios until 2008. The Euro area as a whole could reduce indebtedness from 1999 to 2007 with respect to GDP. However, the situation changed completely after 2008. Debt-to-GDP ratios skyrocketed in all Euro countries. This development is linked to the financial crisis which started in 2008.

Thus, the main reason for the current crisis in Europe is not errors made during the construction of the Euro area. There was no sovereign debt crisis in Europe until 2008. The Euro crisis is mainly a result of 


\begin{tabular}{|c|c|c|c|c|c|c|c|c|c|c|c|c|c|c|c|c|c|c|c|}
\hline 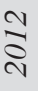 & op & $\begin{array}{l}9 \\
0 \\
\dot{p}\end{array}$ & $\begin{array}{l}20 \\
0 \\
9 \\
1\end{array}$ & $\begin{array}{l}m \\
\varphi_{1}\end{array}$ & $\begin{array}{l}0 \\
0 \\
1\end{array}$ & 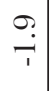 & $\underset{\substack{\infty \\
\dot{1}}}{ }$ & ọ & $\begin{array}{l}0 \\
\dot{0} \\
\overrightarrow{1}\end{array}$ & ب & $\begin{array}{l}0 \\
0 \dot{1}\end{array}$ & 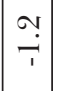 & $\begin{array}{l}\infty \\
0 \\
0 \\
1\end{array}$ & $\begin{array}{l}m \\
m \\
m\end{array}$ & $\overrightarrow{+}$ & $\begin{array}{l}\overrightarrow{1} \\
\dot{1} \\
1\end{array}$ & $\stackrel{m}{\not}$ & $\underset{+}{0}$ & $\begin{array}{l}0 \\
\stackrel{0}{0}\end{array}$ \\
\hline$\underset{\Xi}{\grave{2}}$ & 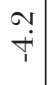 & $\begin{array}{c}1 \\
0 \\
0\end{array}$ & $\begin{array}{l}20 \\
0 \\
1 \\
1\end{array}$ & 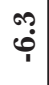 & $\stackrel{\sim}{\sim}$ & $\begin{array}{l}\infty \\
\dot{\varphi} \\
\dot{1}\end{array}$ & $\mid \begin{array}{l}0 \\
1 \\
1 \\
1\end{array}$ & $\begin{array}{l}\infty \\
0 \\
1\end{array}$ & $\mid \begin{array}{l}10 \\
0 \\
1 \\
\end{array}$ & $\begin{array}{c}+ \\
0 \\
7 \\
7\end{array}$ & $\begin{array}{l}\infty \\
0 \\
0 \\
0\end{array}$ & $\begin{array}{l}0 \\
\ddot{1}\end{array}$ & $\begin{array}{l}0 \\
0 \\
0 \\
1\end{array}$ & $\begin{array}{l}\infty \\
\dot{\gamma} \\
\dot{\gamma}\end{array} \mid$ & $\mid$\begin{tabular}{|c|} 
\\
\\
\end{tabular} & $\underset{+}{+}$ & فํ. & $\left|\begin{array}{l}+ \\
0 \\
0 \\
1\end{array}\right|$ & क्. \\
\hline 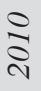 & $\begin{array}{l}0 \\
0 \\
1 \\
1\end{array}$ & $\begin{array}{l}\infty \\
0 \\
0 \\
0\end{array}$ & 年 & $\begin{array}{l}\text { ?ִ } \\
100\end{array}$ & $\begin{array}{c}\Upsilon \\
0 \\
0\end{array}$ & 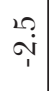 & $\underset{T}{T}$ & 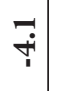 & $\begin{array}{l}r \\
\dot{0} \\
\dot{1}\end{array}$ & $\begin{array}{l}\infty \\
\dot{0} \\
0 \\
0\end{array}$ & $\mid \begin{array}{l}20 \\
\\
\end{array}$ & $\vec{\infty}$ & $\begin{array}{l}0 \\
0 \\
0\end{array}$ & $\begin{array}{c}0 \\
\dot{p} \\
\dot{p}\end{array}$ & : & $\begin{array}{l}\infty \\
\dot{1}\end{array}$ & ri & $\mid \begin{array}{l}\mid \\
\\
\end{array}$ & $\ddot{q}$ \\
\hline 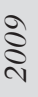 & $\begin{array}{l}+! \\
\stackrel{0}{1}\end{array}$ & $\left|\begin{array}{c|}0 \\
0 \\
1 \\
1\end{array}\right|$ & تُ & ب̂. & $\left|\begin{array}{c}0 \\
\dot{v} \\
\dot{v}\end{array}\right|$ & $\begin{array}{l}20 \\
\stackrel{2}{1} \\
1\end{array}$ & \begin{tabular}{|l|} 
\\
\\
1
\end{tabular} & $\overrightarrow{0}$ & $\left|\begin{array}{c}0 \\
10 \\
10 \\
7\end{array}\right|$ & $\begin{array}{c}0 \\
0 \\
7 \\
7\end{array}$ & $\mid \begin{array}{l}10 \\
10 \\
1\end{array}$ & $\begin{array}{l}\infty \\
\dot{p} \\
\dot{p}\end{array}$ & $\left|\begin{array}{l}\infty \\
\dot{0} \\
1\end{array}\right|$ & pi & ק. & 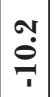 & $\begin{array}{l}0 \\
\infty \\
1\end{array}$ & $\left|\begin{array}{c}\mathscr{y} \\
\dot{0}\end{array}\right|$ & ִִ \\
\hline $\begin{array}{l}\infty \\
\stackrel{2}{2} \\
\sim\end{array}$ & $\overrightarrow{q_{i}}$ & $\left|\begin{array}{l}0 \\
-i\end{array}\right|$ & $\begin{array}{l}0 \\
0 \\
0 \\
1\end{array} \mid$ & $\ddot{0}$ & $\left|\begin{array}{l}\sigma \\
\sigma \\
\sigma_{1}\end{array}\right|$ & $\stackrel{+}{+}$ & $\begin{array}{c}0 ? \\
0 \\
0\end{array}$ & $\overrightarrow{0}$ & $\begin{array}{l}\infty \\
\dot{p}\end{array}$ & 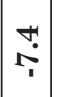 & $\begin{array}{l}- \\
\sigma_{1}\end{array}$ & $\begin{array}{l}\text { ָy } \\
\underset{1}{ }\end{array}$ & $\mid \begin{array}{l}0 y \\
\text { or }\end{array}$ & $\left|\begin{array}{c}0 \\
\dot{T}\end{array}\right|$ & $\overbrace{}^{20}$ & $\begin{array}{l}0 \\
\dot{0}\end{array}$ & $\overrightarrow{\sigma_{1}}$ & 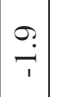 & "ִ \\
\hline$\stackrel{\curvearrowright}{\curvearrowright}$ & $\begin{array}{l}\hat{0} \\
\dot{0}\end{array}$ & $\overrightarrow{0}$ & $\begin{array}{l}0 \\
0 \\
0\end{array}$ & $\begin{array}{l}20 \\
08\end{array}$ & $\begin{array}{c}\vec{H} \\
\stackrel{v}{ }\end{array}$ & $\begin{array}{l}\infty \\
100\end{array}$ & $\vec{v}$ & $\stackrel{o r}{0}$ & $\mid \begin{array}{l}20 \\
0 \\
0\end{array}$ & $\overrightarrow{0}$ & $\begin{array}{l}0 \\
-1\end{array}$ & $\begin{array}{l}+ \\
\dot{1} \\
\dot{1}\end{array}$ & $\overrightarrow{0}$ & $\begin{array}{l}\infty \\
\tilde{q} \\
\dot{q}\end{array}$ & ọ & के & 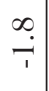 & $\begin{array}{l}0 \\
0\end{array}$ & ? \\
\hline $\begin{array}{l}๖ \\
\check{2}\end{array}$ & $\stackrel{n}{1}$ & $\stackrel{t}{0}$ & $\stackrel{20}{7}$ & 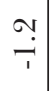 & $\begin{array}{l}20 \\
0 \mathrm{v}\end{array}$ & $\underset{\sim}{\stackrel{y}{*}}$ & $\begin{array}{l}0 \\
\tilde{q} \\
\tilde{q}\end{array}$ & $\stackrel{0}{\rightarrow}$ & 19: & $\begin{array}{l}\sigma \\
\dot{v}\end{array} \mid$ & $\begin{array}{l}+\vdots \\
0 \\
0\end{array}$ & \begin{tabular}{|c|}
20 \\
0 \\
1
\end{tabular} & $\stackrel{\leftrightarrow}{\sim}$ & $\vec{\sim}$ & 10 & & $\begin{array}{l}\text { ọ } \\
\text { ọ }\end{array}$ & $\stackrel{+}{+}$ & $\begin{array}{l}\vec{H} \\
\stackrel{v}{ }\end{array}$ \\
\hline $\begin{array}{l}i n \\
\stackrel{2}{2} \\
\curvearrowright\end{array}$ & $\begin{array}{l}20 \\
\sigma i \\
\gamma\end{array}$ & $\left|\begin{array}{c}20 \\
20 \\
i \\
1\end{array}\right|$ & $\stackrel{\sim}{-}$ & $\begin{array}{c}\stackrel{+}{0} \\
\stackrel{i}{i}\end{array}$ & $\stackrel{0}{-}$ & $\begin{array}{l}o \\
\sigma\end{array}$ & $\begin{array}{l}0 \\
\dot{\gamma} \\
\dot{1}\end{array}$ & ?ִ & $\begin{array}{l}\text { ȳ } \\
\text { ip }\end{array}$ & 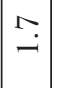 & म̦. & $\begin{array}{l}+ \\
\dot{0} \\
1\end{array}$ & 0 & $\begin{array}{l}0 \\
\dot{\gamma}\end{array}$ & ? & "ִ & $\begin{array}{l}\infty \\
o \\
i\end{array}$ & רי & $\stackrel{?}{\longrightarrow}$ \\
\hline 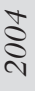 & $\begin{array}{l}\stackrel{\sigma}{0} \\
\stackrel{v}{1}\end{array}$ & $\overrightarrow{\dot{\varphi}}$ & $\begin{array}{l}+ \\
\\
\end{array}$ & $\underset{ن}{\overrightarrow{+}}$ & $\stackrel{0}{-}$ & $\begin{array}{l}20 \\
0 \\
0\end{array}$ & $\left|\begin{array}{c}0 \\
\dot{p}\end{array}\right|$ & pa & \begin{tabular}{|l|}
10 \\
\\
\end{tabular} & $\stackrel{\leftrightarrow}{-}$ & ?ח & $\begin{array}{l}0 \\
-1 \\
1\end{array}$ & $\stackrel{\longrightarrow}{\longrightarrow}$ & $\begin{array}{l}0 \\
\dot{+}\end{array}$ & $\stackrel{\sim}{r}$ & $\stackrel{\ominus}{+}$ & $\begin{array}{c}\vec{\forall} \\
\vec{\gamma} \\
1\end{array}$ & $\begin{array}{l}0 \\
\tilde{\gamma} \\
\tilde{q}\end{array} \mid$ & $\overrightarrow{\dot{\varphi}}$ \\
\hline 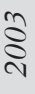 & $\vec{p}$ & $\vec{\varphi}$ & דִ & $\begin{array}{l}0 \\
\dot{\varphi} \\
1\end{array}$ & $\stackrel{\sim}{\sim}$ & $\begin{array}{l}0 \\
\dot{v}\end{array}$ & تُ & $\begin{array}{c}\mathscr{y} \\
\underset{1}{1}\end{array}$ & $\begin{array}{c}0 \\
10 \\
10\end{array}$ & $\stackrel{+}{\circ}$ & $\begin{array}{c}0 \\
0 \\
0 \\
\dot{p}\end{array}$ & $\left|\begin{array}{l}0 \\
-1\end{array}\right|$ & ?20 & $\begin{array}{l}0 \\
0 \\
0\end{array}$ & $\rightarrow$ & $\vec{p}$ & $\begin{array}{c}\infty \\
\dot{\gamma} \\
1\end{array}$ & $\begin{array}{l}r \\
\dot{v}\end{array}$ & $\stackrel{\infty}{p}$ \\
\hline 8 & $\begin{array}{c}0 \\
\underset{v}{1}\end{array}$ & $\overrightarrow{0}$ & $\begin{array}{l}1 \\
\dot{0}\end{array}$ & $\underset{+}{+}$ & ?ִ & $\stackrel{\sim}{\sim} \underset{+}{ }$ & $\overrightarrow{0}$ & $\stackrel{\infty}{\infty}$ & $\underset{\substack{\infty \\
\dot{T}}}{ }$ & $\stackrel{+}{\dot{0}}$ & 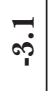 & $\begin{array}{l}o \\
o j \\
q \\
1\end{array}$ & $\vec{a}$ & : & $\overrightarrow{\vec{v}}$ & $\begin{array}{c}+! \\
0 \\
0\end{array}$ & $\mid \begin{array}{c}\text { vy } \\
\infty \\
1\end{array}$ & $\begin{array}{l}\overrightarrow{+} \\
\dot{v}\end{array}$ & $\underset{1}{\stackrel{0}{0}}$ \\
\hline$\underset{\delta}{2}$ & $\stackrel{0}{-1}$ & $\stackrel{H}{0}$ & $\stackrel{0}{0}$ & $\begin{array}{l}q \\
o v \\
v i\end{array}$ & $\begin{array}{l}\overrightarrow{0} \\
1\end{array}$ & 70 & $\mid$ & $\overrightarrow{0}$ & 10 & $\ddot{0}$ & $\overrightarrow{0}$ & $\left|\begin{array}{c}0 \\
\dot{\gamma} \\
1\end{array}\right|$ & $\overrightarrow{0}$ & $\begin{array}{l}0 \\
0 \\
0 \\
1\end{array}$ & ơ & 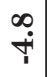 & $\left|\begin{array}{l}10 \\
0 \\
1\end{array}\right|$ & $\stackrel{0}{+}$ & ?ִ \\
\hline 8 & $\overrightarrow{\dot{\varphi}}$ & $\stackrel{0}{0}$ & $\stackrel{r}{r}$ & $\begin{array}{l}m \\
\ddot{v}\end{array}$ & $\begin{array}{c}0 \\
0 \\
0 \\
1\end{array}$ & $\stackrel{0}{0}$ & ?ִ & $\rightleftarrows$ & $\vec{p}$ & $\dot{F}$ & $\stackrel{\infty}{\infty}$ & $\left|\begin{array}{l}\infty \\
\dot{\gamma}\end{array}\right|$ & 0 & $\begin{array}{l}-1 \\
10\end{array}$ & $\begin{array}{c}0 \\
\dot{v}\end{array}$ & ?ִ & 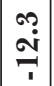 & $\vec{r}$ & $\stackrel{0}{0}$ \\
\hline ஓे & חִ & $\begin{array}{l}0 \\
\dot{\varphi}\end{array}$ & $\begin{array}{l}\infty \\
q \\
q\end{array}$ & $\frac{\infty}{+}$ & ?ִ & $\stackrel{\sim}{-}$ & 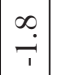 & $\begin{array}{l}0 \\
-1 \\
-1\end{array}$ & & $\begin{array}{c}0 \\
\dot{v}\end{array}$ & $\stackrel{0}{T}$ & $\begin{array}{l}0 \\
0 \\
0\end{array}$ & r. & $\begin{array}{l}0 \\
\dot{1} \\
\dot{1}\end{array}$ & $\stackrel{+}{0}$ & i. & 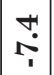 & $\begin{array}{c}0 \\
0 \\
0\end{array}$ & ִִ \\
\hline$=$ & 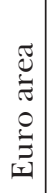 & 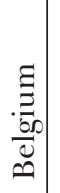 & 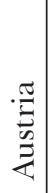 & 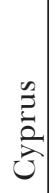 & 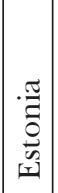 & $\underset{\Xi}{\stackrel{\Xi}{\Xi}}$ & 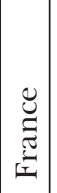 & 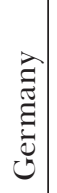 & 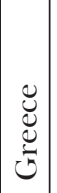 & 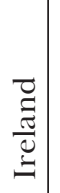 & $\stackrel{\vec{\Xi}}{\Xi}$ & 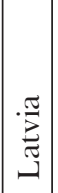 & 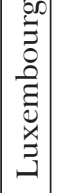 & $\frac{\tilde{J}}{\sum^{\frac{\pi}{\Sigma}}}$ & 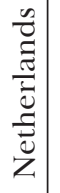 & 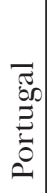 & $\left|\begin{array}{l}\frac{\pi}{\pi} \\
\frac{\pi}{\pi} \\
\frac{0}{\sim}\end{array}\right|$ & $\begin{array}{c}\cdot \frac{\pi}{J} \\
\bar{d} \\
\frac{\partial}{n}\end{array} \mid$ & 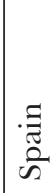 \\
\hline
\end{tabular}




\begin{tabular}{|c|c|c|c|c|c|c|c|c|c|c|c|c|c|c|c|c|c|c|c|}
\hline$\frac{c}{8}$ & $\begin{array}{l}0 \\
\dot{8}\end{array}$ & $\stackrel{0}{\dot{\sigma}}$ & $\begin{array}{l}+ \\
\stackrel{\nabla}{r}\end{array}$ & $\begin{array}{l}\infty \\
10 \\
\infty\end{array}$ & $\ddot{0}$ & $\begin{array}{c}0 \\
00 \\
i 0\end{array}$ & $\begin{array}{l}\text { ช̛ } \\
\text { \&̊ }\end{array}$ & 官 & $\begin{array}{l}0 \\
0 \\
0 \\
20 \\
-1\end{array}$ & $\begin{array}{l}0 \\
\\
\end{array}$ & $\begin{array}{c}0 \\
\mathbf{N} \\
\stackrel{N}{u}\end{array} \mid$ & $\begin{array}{l}1 \\
\dot{q} \\
+\end{array}$ & $\begin{array}{l}\infty \\
\stackrel{\dot{\theta}}{\sigma}\end{array}$ & 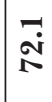 & 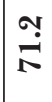 & 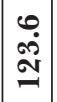 & $\begin{array}{l}\vec{v} \\
20\end{array}$ & تُ & $\underset{\sim}{\widetilde{D}}$ \\
\hline $\bar{c}$ & $\begin{array}{l}\infty \\
\infty \\
\infty\end{array}$ & $\begin{array}{l}\infty \\
\stackrel{\infty}{\sigma}\end{array}$ & $\begin{array}{l}20 \\
\mathfrak{N} \\
\stackrel{1}{N}\end{array}$ & $ت$ & $\begin{array}{l}0 y \\
0\end{array}$ & $\begin{array}{l}0 \\
\dot{\sigma}\end{array}$ & $\begin{array}{l}\infty \\
10 \\
\infty\end{array}$ & $\begin{array}{l}+1 \\
\dot{\infty} \\
\infty\end{array}$ & 足 & $\begin{array}{l}+ \\
\vdots \\
0 \\
0\end{array}$ & $\mid \begin{array}{l}\infty \\
\dot{\sim} \\
\stackrel{N}{ }\end{array}$ & $\frac{9}{\dot{q}}$ & $\begin{array}{l}\infty \\
\infty \\
\infty\end{array}$ & $\stackrel{m}{0}$ & \begin{tabular}{|l}
20 \\
30 \\
0
\end{tabular} & $\begin{array}{c}\infty \\
\infty \\
0 \\
0\end{array}$ & $\begin{array}{l}\infty \\
\infty \\
+\infty \\
\end{array}$ & $\begin{array}{l}\mathscr{\rho} \\
\dot{1}\end{array}$ & $\ddot{8}$ \\
\hline 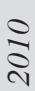 & $\begin{array}{l}+4 \\
i \\
\infty\end{array}$ & $\begin{array}{l}10 \\
10 \\
0\end{array}$ & $\begin{array}{c}0 \\
\stackrel{N}{N}\end{array}$ & ?ִ & $\begin{array}{l}1 \\
0\end{array}$ & $\begin{array}{l}0 \\
\dot{\infty} \\
\dot{f}\end{array}$ & $\begin{array}{l}\vec{t} \\
\dot{\alpha} \\
\infty\end{array}$ & $\begin{array}{c}\forall \vdots \\
\ddot{\alpha} \\
\infty\end{array}$ & \begin{tabular}{|c|}
$\infty$ \\
$\infty$ \\
$\infty$ \\
+ \\
\end{tabular} & $\overrightarrow{\widehat{\sigma}}$ & 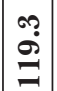 & $\begin{array}{l}+ \\
\dot{f}\end{array}$ & 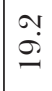 & 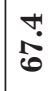 & 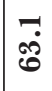 & $\begin{array}{l}\stackrel{0}{\dot{H}} \\
\text { ô }\end{array}$ & $\stackrel{0}{\underset{7}{+}}$ & $\begin{array}{l}0 \\
\infty \\
\infty\end{array}$ & $\frac{20}{60}$ \\
\hline$\stackrel{\overbrace{}}{\check{\vartheta}}$ & $\begin{array}{l}\dot{0} \\
\dot{\infty}\end{array}$ & 10 & 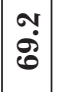 & $\begin{array}{l}0 \\
10\end{array}$ & ọ & $\begin{array}{l}20 \\
\rightarrow \\
\rightarrow\end{array}$ & 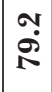 & 足 & $\begin{array}{l}\tilde{D} \\
\stackrel{2}{2}\end{array}$ & $\begin{array}{l}\infty \\
\dot{+} \\
\dot{0}\end{array}$ & $\begin{array}{l}\forall \\
0 \\
\Xi\end{array}$ & $\begin{array}{l}0 \\
\dot{0} \\
0\end{array} \mid$ & $\begin{array}{l}\infty \\
20 \\
20\end{array}$ & ¿. & $\begin{array}{l}\infty \\
\dot{8}\end{array}$ & $\begin{array}{l}\square \\
\infty \\
\infty\end{array}$ & $\begin{array}{c}0 \\
20 \\
00\end{array} \mid$ & $\begin{array}{c}0 \\
20 \\
00\end{array}$ & $\begin{array}{l}0 \\
0 \\
10\end{array}$ \\
\hline$\underset{\sim}{\infty}$ & 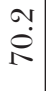 & 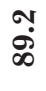 & $\left|\begin{array}{l}\infty \\
\dot{0} \\
0 \\
0\end{array}\right|$ & $\begin{array}{l}0 \\
\infty \\
+ \\
+\end{array}$ & $\stackrel{10}{+1}$ & $\begin{array}{l}\sigma \\
\infty \\
\infty \\
\infty\end{array}$ & $\begin{array}{l}0 \\
0 \\
0 \\
0\end{array}$ & $\left|\begin{array}{l}\infty \\
0 \\
0 \\
0\end{array}\right|$ & $\begin{array}{l}\stackrel{\sigma}{a} \\
\stackrel{\sim}{\Xi} \\
\beth\end{array}$ & $\begin{array}{l}10 \\
\dot{H} \\
\end{array}$ & $\mid \begin{array}{c}-1 \\
\dot{0} \\
0\end{array}$ & $\begin{array}{l}\infty \\
\dot{g} \\
0\end{array}$ & $\stackrel{+}{+}$ & o & \begin{tabular}{|l}
20 \\
00 \\
10
\end{tabular} & $\underset{1}{1}$ & 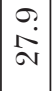 & $\begin{array}{l}0 \\
\dot{\mathcal{N}} \\
\text { v }\end{array}$ & ơ \\
\hline 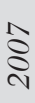 & $\begin{array}{l}+ \\
0 \\
0\end{array}$ & $\underset{\infty}{\stackrel{\infty}{+}}$ & 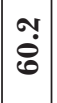 & $\begin{array}{l}\infty \\
\infty \\
10 \\
10\end{array}$ & $\begin{array}{l}1 \\
\infty\end{array}$ & $\begin{array}{l}\text { O } \\
20 \\
\text { on }\end{array}$ & $\begin{array}{l}\text { Y̛ } \\
\text { Jే }\end{array}$ & 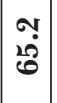 & 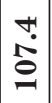 & $\begin{array}{l}-7 \\
20 \\
\stackrel{2}{N}\end{array}$ & $\begin{array}{c}m \\
0 ? \\
\tilde{\theta} \\
0\end{array}$ & $\begin{array}{l}0 \\
0\end{array}$ & 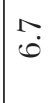 & $\ddot{8}$ & $\begin{array}{l}\infty ? \\
20 \\
7\end{array}$ & \begin{tabular}{|l|}
$\nabla$ \\
$\infty$ \\
0 \\
0
\end{tabular} & $\begin{array}{l}0 \\
\dot{D} \\
\vec{D}\end{array}$ & $\vec{\sim}$ & $\begin{array}{l}\infty \\
0 \\
\text { on }\end{array}$ \\
\hline 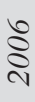 & $\begin{array}{l}0 \\
\dot{0} \\
0\end{array}$ & $\underset{\infty}{\infty}$ & 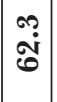 & تُ & $\stackrel{+t}{+}$ & $\begin{array}{l}0 \\
\dot{\rho} \\
\ddot{m}\end{array}$ & $\begin{array}{l}1 \\
\dot{0} \\
0\end{array}$ & $\begin{array}{l}0 \\
0 \\
0 \\
0\end{array}$ & 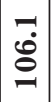 & $\begin{array}{c}0 \\
\dot{+} \\
\sigma\end{array}$ & $\begin{array}{l}n \\
\mathscr{0} \\
0 \\
0\end{array}$ & $\ddot{0}$ & 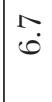 & ช̊ & 莗 & $\begin{array}{l}\forall \\
\dot{\theta}\end{array}$ & $\begin{array}{c}10 \\
0 \\
0 \\
0\end{array}$ & $\underset{\stackrel{\leftrightarrow}{*}}{+}$ & $\stackrel{1}{\dot{\rho}}$ \\
\hline$\stackrel{n}{8}$ & $\stackrel{\infty}{?}$ & $\begin{array}{l}\text { ํ. } \\
\text { જ̛ }\end{array}$ & พ̛̃ & $\begin{array}{c}+1 \\
\dot{0}\end{array}$ & $\begin{array}{l}0 \\
+ \\
+\end{array}$ & $\begin{array}{l}\square \\
\dot{y}\end{array}$ & $\mid \begin{array}{l}4 \\
\dot{0}\end{array}$ & $\mid \begin{array}{l}10 \\
00 \\
0\end{array}$ & $\begin{array}{l}0 \\
\dot{8} \\
\end{array}$ & $\begin{array}{l}n \\
\hat{a} \\
\text { ov }\end{array}$ & $\begin{array}{l}r \\
20 \\
0\end{array}$ & $\begin{array}{l}20 \\
01 \\
-1\end{array}$ & $\ddot{0}$ & $\begin{array}{l}0 \\
\infty \\
0\end{array}$ & $\frac{\infty}{20}$ & $\begin{array}{l}1 \\
0 \\
0\end{array}$ & $\begin{array}{c}o \\
\stackrel{1}{*} \\
\text { on }\end{array}$ & ज् & $\begin{array}{l}\text { o } \\
\text { on } \\
\text { f }\end{array}$ \\
\hline$\underset{\overbrace{}}{\stackrel{D}{๖}}$ & $\begin{array}{l}0 \\
\dot{8}\end{array}$ & $\stackrel{\circ}{\circ}$ & تีं & 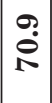 & $\left|\begin{array}{c}0 \\
10\end{array}\right|$ & $\underset{+}{+}$ & Oें & 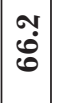 & $\begin{array}{c}0 \\
\dot{0} \\
\mathscr{\sigma}\end{array}$ & $\begin{array}{l}20 \\
2 \\
2 \\
2\end{array}$ & $\begin{array}{c}1 \\
\dot{\theta} \\
\dot{\sigma}\end{array}$ & $\begin{array}{c}0 \\
10 \\
-1\end{array}$ & $0 ?$ & 我 & $\begin{array}{l}\vec{H} \\
\stackrel{2}{\mathcal{N}} \\
20\end{array}$ & $\frac{\sigma}{6}$ & $\stackrel{20}{7}$ & $\begin{array}{l}\text { on } \\
\text { N }\end{array}$ & $\stackrel{\infty}{\mathscr{0}}$ \\
\hline$\stackrel{n}{\check{\vartheta}}$ & 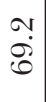 & $\begin{array}{l}+1 \\
\infty \\
\infty\end{array}$ & $\begin{array}{l}m \\
20 \\
0\end{array}$ & $\ddot{8}$ & $\begin{array}{c}0 \\
20\end{array}$ & $\stackrel{10}{+}$ & $\begin{array}{l}\boldsymbol{\sigma} \\
\dot{\mathcal{U}} \\
\boldsymbol{U}\end{array}$ & 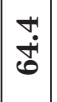 & 节 & $\begin{array}{l}1 \\
\dot{0} \\
0 \\
\text { on }\end{array}$ & 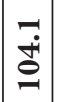 & $\stackrel{I}{ \pm}$ & $\overrightarrow{0}$ & ○्. & $\begin{array}{l}0 \\
\dot{N} \\
20\end{array}$ & $\begin{array}{l}\forall \\
\sigma \\
2\end{array}$ & $\begin{array}{l}\stackrel{H}{a} \\
\stackrel{v}{H}\end{array}$ & ণ & $\begin{array}{l}\infty \\
\stackrel{\infty}{+} \\
+\end{array}$ \\
\hline$\stackrel{\overbrace{}}{\check{\overbrace{}}}$ & $\begin{array}{l}0 \\
\infty \\
0\end{array}$ & 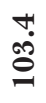 & 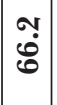 & 70 & $\begin{array}{l}1 \\
20\end{array}$ & 号 & \begin{tabular}{|l|}
$\infty$ \\
$\infty$ \\
10 \\
10
\end{tabular} & $\ddot{\dot{\theta}}$ & 형 & $\begin{array}{c}0 \\
\text { ov } \\
\text { on }\end{array}$ & $\begin{array}{l}7 \\
10 \\
0 \\
-1\end{array}$ & $\begin{array}{c}0 \\
0 \dot{2}\end{array}$ & ? & $\begin{array}{l}0 \\
0 \\
\text { in }\end{array}$ & \begin{tabular}{|l}
10 \\
80 \\
10
\end{tabular} & $\left|\begin{array}{l}\infty \\
0 \\
20\end{array}\right|$ & $\begin{array}{c}\vec{t} \\
\dot{\infty} \\
+\end{array}$ & $\stackrel{\infty}{N}$ & $\begin{array}{l}0 \\
\text { o } \\
1\end{array}$ \\
\hline$\underset{\overbrace{}}{8}$ & $\begin{array}{l}y \\
0 \\
0 \\
0\end{array}$ & مُ & $\left|\begin{array}{l}\infty \\
0 \\
0\end{array}\right|$ & 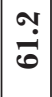 & $\begin{array}{l}\infty \\
+ \\
\dot{i}\end{array}$ & $\begin{array}{l}20 \\
\stackrel{y}{y} \\
\stackrel{y}{2}\end{array}$ & $\left|\begin{array}{l}0 \\
0 \\
20\end{array}\right|$ & $\begin{array}{l}7 \\
2 \\
20\end{array}$ & $\begin{array}{c}- \\
\dot{\theta} \\
\varrho\end{array}$ & $\begin{array}{c}o y \\
10 \\
00\end{array}$ & $\begin{array}{l}\infty \\
\infty \\
\infty \\
0 \\
0\end{array}$ & $\underset{+}{ \pm}$ & ?ִ & $\begin{array}{l}0 \\
0 \\
\infty \\
20\end{array}$ & $\begin{array}{l}1 \\
0 \\
20\end{array}$ & $\begin{array}{l}\infty \\
0 \\
i 0 \\
10\end{array}$ & $\begin{array}{l}0 \\
\infty \\
+\infty\end{array} \mid$ & $\begin{array}{l}20 \\
\stackrel{0}{0}\end{array}$ & $\begin{array}{l}0 \\
10 \\
10\end{array}$ \\
\hline$\S$ & 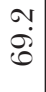 & $\stackrel{\infty}{\stackrel{\infty}{0}} \underset{0}{0}$ & 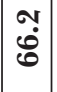 & $\begin{array}{l}0 \\
0 \\
10 \\
10\end{array}$ & 7 & $\begin{array}{l}\infty \\
\infty \\
\dot{7}\end{array}$ & $\begin{array}{l}0 \\
10 \\
10\end{array}$ & 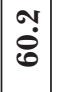 & $\begin{array}{c}+1 \\
\dot{0} \\
0 \\
\sigma\end{array}$ & $\begin{array}{c}70 \\
20 \\
00\end{array}$ & $\left|\begin{array}{c}0 \\
0 \\
0 \\
0 \\
0\end{array}\right|$ & 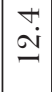 & ơ & $\begin{array}{l}0 \\
00 \\
10\end{array}$ & $\begin{array}{l}\infty \\
i 0 \\
10\end{array}$ & $\begin{array}{l}1 \\
\dot{8} \\
20\end{array}$ & $\begin{array}{l}n \\
0 \\
10\end{array}$ & $\begin{array}{l}\infty \\
\stackrel{0}{0}\end{array}$ & $\begin{array}{l}+ \\
\text { बे }\end{array}$ \\
\hline ڤे & $\stackrel{i}{\Sigma}$ & $\stackrel{0}{\stackrel{0}{g}}$ & $\begin{array}{l}\infty \\
0 \\
0 \\
0\end{array}$ & $\begin{array}{l}\infty \\
0 \\
20 \\
20\end{array}$ & \begin{tabular}{|l|}
20 \\
0
\end{tabular} & $\begin{array}{l}r \\
20 \\
\text { fi }\end{array}$ & $\left|\begin{array}{l}0 \\
\infty \\
10\end{array}\right|$ & $\stackrel{m}{\sigma}$ & 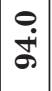 & \begin{tabular}{|c|}
0 \\
1 \\
\\
\end{tabular} & a & 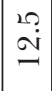 & $\stackrel{+}{0}$ & $\begin{array}{l}9 \\
10 \\
10\end{array}$ & 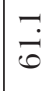 & $\frac{+}{\dot{2}}$ & $\begin{array}{l}\infty \\
\dot{x} \\
\dot{\gamma}\end{array}$ & $\underset{d}{\vec{d}}$ & $\begin{array}{l}\text { ர் } \\
\text { ஸ் }\end{array}$ \\
\hline है & 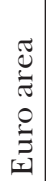 & 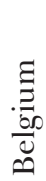 & 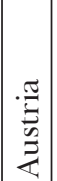 & 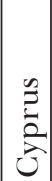 & 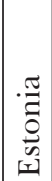 & 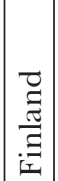 & 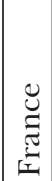 & 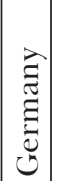 & 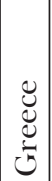 & 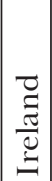 & $\frac{\lambda}{\Xi}$ & 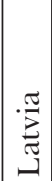 & 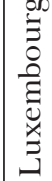 & $\frac{\stackrel{\pi}{J}}{\underline{J}}$ & 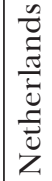 & 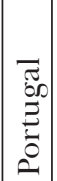 & 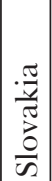 & $\begin{array}{l}\frac{\pi}{J} \\
\frac{0}{d} \\
\frac{0}{n}\end{array}$ & $\begin{array}{l}\text { Iี } \\
\text { ڤิ }\end{array}$ \\
\hline
\end{tabular}


the subprime crisis that started in 2007 in the US. This inevitably leads to the question why Europe was affected so severely. Many European banks invested in Asset Backed Securities (ABS). After 2001 monetary policy in Europe was relatively expansionary. ${ }^{4}$ This led banks and investors to focus on alternative investments as traditional bonds were not lucrative due to low yields. ABs seemed to be a reasonable option as they obtained consistently good ratings and had higher yields. Therefore, banks could finance themselves cheaply at the ЕСв and buy high-yield ABS. The other reason for the involvement of Europe in the Subprime sector via ABS was that the us had large current account deficits which it needed to finance. The us trade deficits were financed by selling ABS to countries with current account surpluses, to Germany and the Netherlands in particular. When the Federal Reserve System (FED) increased interest rates from 2006 to 2007, the housing bubble in the us burst and ABs plummeted in value. This triggered massive write-downs of banks' assets and, indeed, created mistrust between banks and between depositors and banks. Consequently, depositors withdrew money from banks and banks were loath to lend money to one another. However, the situation was still relatively calm up to the point when Lehman Brothers failed. This insolvency caused a crash of stock markets worldwide and led to further write-downs and bank losses. This triggered solvency and liquidity problems for many banks.

The difference between continental Europe and Anglo-Saxon countries is that in the latter the financial system is market-orientated and not bank dominated. In Europe, companies and individuals finance investments mainly by borrowing money from their house banks and retaining profits. Small and medium enterprises rarely issue corporate bonds to obtain liquidity. Thus, banks are essential for the functioning of the real economy. Furthermore, domestic banks are relatively large compared to the size of the domestic economy. ${ }^{5}$ Several banks' balance sheet totals account for more than $100 \%$ of the GDP of the country that they are

${ }^{4}$ Between June 2003 and November 2005 the interest rate for main refinancing operations was $2 \%$.

${ }^{5}$ The ratio of bank assets to GDP is 16 for Luxembourg, 6 for Ireland, 4 for the Netherlands, and around 3.5 for Spain and Germany (Data from June 2001; see Schoenmaker and Werkhoven, 2012). 
located in. ${ }^{6}$ Moreover, European banks still mainly invest in domestic assets and government bonds. This creates mutual dependency between banks and governments. Banks' solvency depends on the solvency of their home country and vice versa. This dependence forced European governments to rescue banks. Many banks had to be restructured or needed capital and liquidity. Figure 1 shows the amount of rescue measures in the Euro area.

As figure 1 illustrates, rescue measures consisted of guarantees, relief measures, recapitalisation measures, and liquidity measures. Guarantees account for the largest part. In 2008, these amounted to 320 billion Euros. In 2009, the sum was more than twice as high as in 2008. The same applies for 2010. It is also instructive to take a look at the number of restructured banks between 2008 and 2010. Italy (19) and Austria (17) restructured most banks during that time. This is still much lower than the number of restructured banks in the us (53). Most interestingly, Spain

Figure 1. Expenditures of Euro countries for bank rescue operations

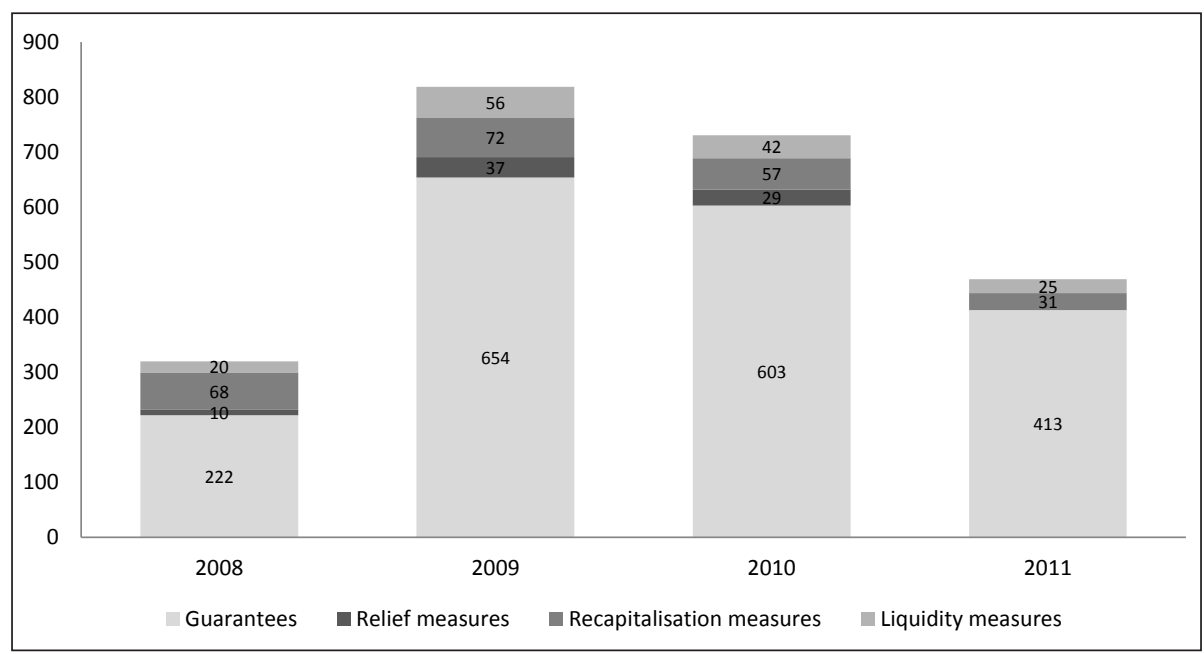

Source: European Commission, Demary and Schuster, 2013.

${ }^{6}$ This applies for ING, Santander, and BNP Paribas (International Monetary Fund [IMF], 2013). 
closed only three banks. ${ }^{7}$ Spain is one of the countries which is most affected by the crisis because it also suffered from a burst domestic housing price bubble. Thus, one would expect that Spain would restructure many more banks, especially because there are lots of distressed small thrifts (e.g. cajas de ahorro). But why did Spain close only three banks? There are basically two reasons. First, politicians were acutely aware of the problems of the housing market. Closing more banks would have put more pressure on prices due to higher foreclosure rates. They also wanted that banks keep lending money to the private sector to stabilize the economy. Second, the government was aware of massive fiscal costs that a restructuring would have incurred. Thus, they hesitated to clear up the banking sector. In retrospect, we know that the delay of the banking crisis was a huge mistake. Countries like the us that cleaned up the mess quickly, are now in a much better situation.

However, the main reason for the current sovereign debt crisis is excessive private debt and not public debt. Banks and individuals increased lending. The problem was that these credits were not only used for productivity increasing investments but for consumption and investment in real estate.

Figure 2 shows the development of private debt in selected Euro zone countries. Private debt was apparently on an expansion path until the beginning of the financial crisis. Only some countries managed to keep private debt as a percentage of GDP stable after the breakout of the crisis. Spending by individuals, companies, and governments in the PIIGS countries were financed by capital account surpluses (see section "The Balance of Payment Crisis"). Lending was used for housing purchases. At the peak of the housing boom the proportion of building investments with respect to GDP was 22\% in Ireland and $18 \%$ in Spain (Neubäumer 2011, p. 828). Spain and Ireland had their own housing price bubbles. These bubbles burst shortly after the subprime mortgage bubble.

To summarize, the root of the evil lies in the financial crisis. Before the outbreak of the financial crisis, the Euro zone managed to cope with problems which went back to the founding of the currency area. The Great Recession forced countries to rescue banks and companies and to conduct expansionary fiscal policy to fight the recession.

${ }^{7}$ Data is taken from Demary and Schuster (2013). 


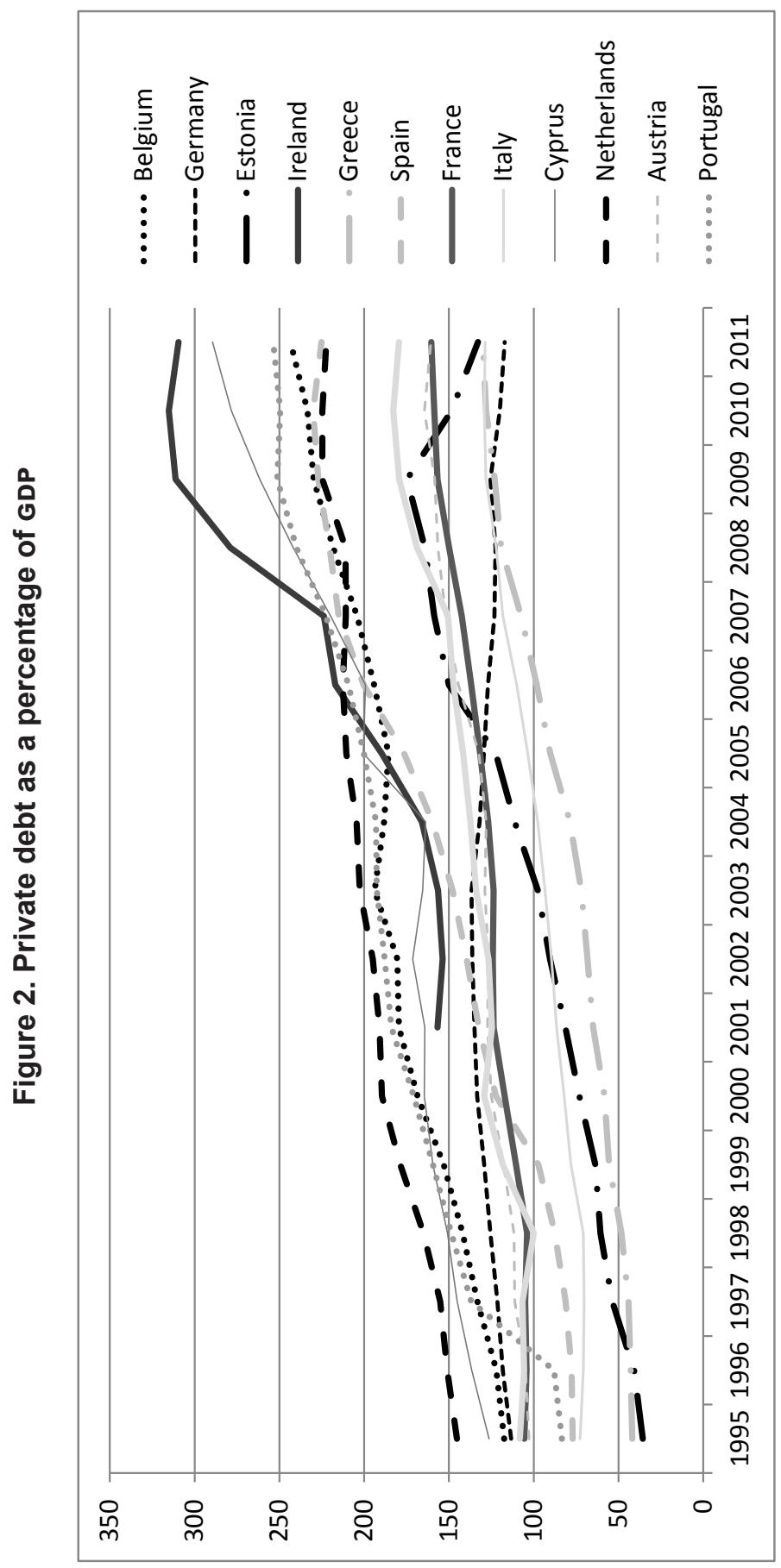

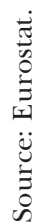




\section{Outlining the Euro crisis}

After discussing the causes of the Euro crisis, the next section will show that the current crisis has not just one symptom (sovereign debt) but a multiplicity. The four major crises are the growth crisis, the labour market crisis, the sovereign debt crisis, and the balance of payment crisis which will be discussed in this chapter.

\section{The growth crisis}

The Euro zone is not just in a sovereign debt crisis, it is also in a growth crisis. The financial crisis led to a recession of the entire Euro zone in 2009. All members were affected by this crisis. Figure 3 shows real GDP growth rates of selected countries as well as the average among all Euro countries. The figure demonstrates that the euro zone was hit by a doubledip recession, a typical feature of many banking crises.

Before the financial crisis started, Germany was "the sick man of Europe" as growth rates were mostly well below the average of the euro zone. Then the picture changed dramatically. Germany is the stabilizing pillar of the Euro zone economy. Without Germany the euro zone would probably have been in a recession in 2010 and 2011 as well. While Germany recovered from the financial crisis, other countries are still stuck in a recession. But why is Europe so divided? There are several reasons for this. The first comes from Keynesian theory. Countries with public debt problems opted for or were forced to adopt austerity measures. Consequently, public expenditures were cut and taxes increased. In contradiction to the Ricardian equivalence theorem, government spending cuts were multiplied, widening and deepening the recession. Recession forced governments into further austerity measures, which only made matters worse. At the same time, monetary policy was not effective, as banks were not willing to extend credit to companies and investors because they are still tottering. Second, inflation and wage increase differentials caused different developments in competiveness. Southern states with higher inflation rates lost price competiveness against northern states like Germany, resulting in current account imbalances (see section “The Balance of Payment Crisis”). 


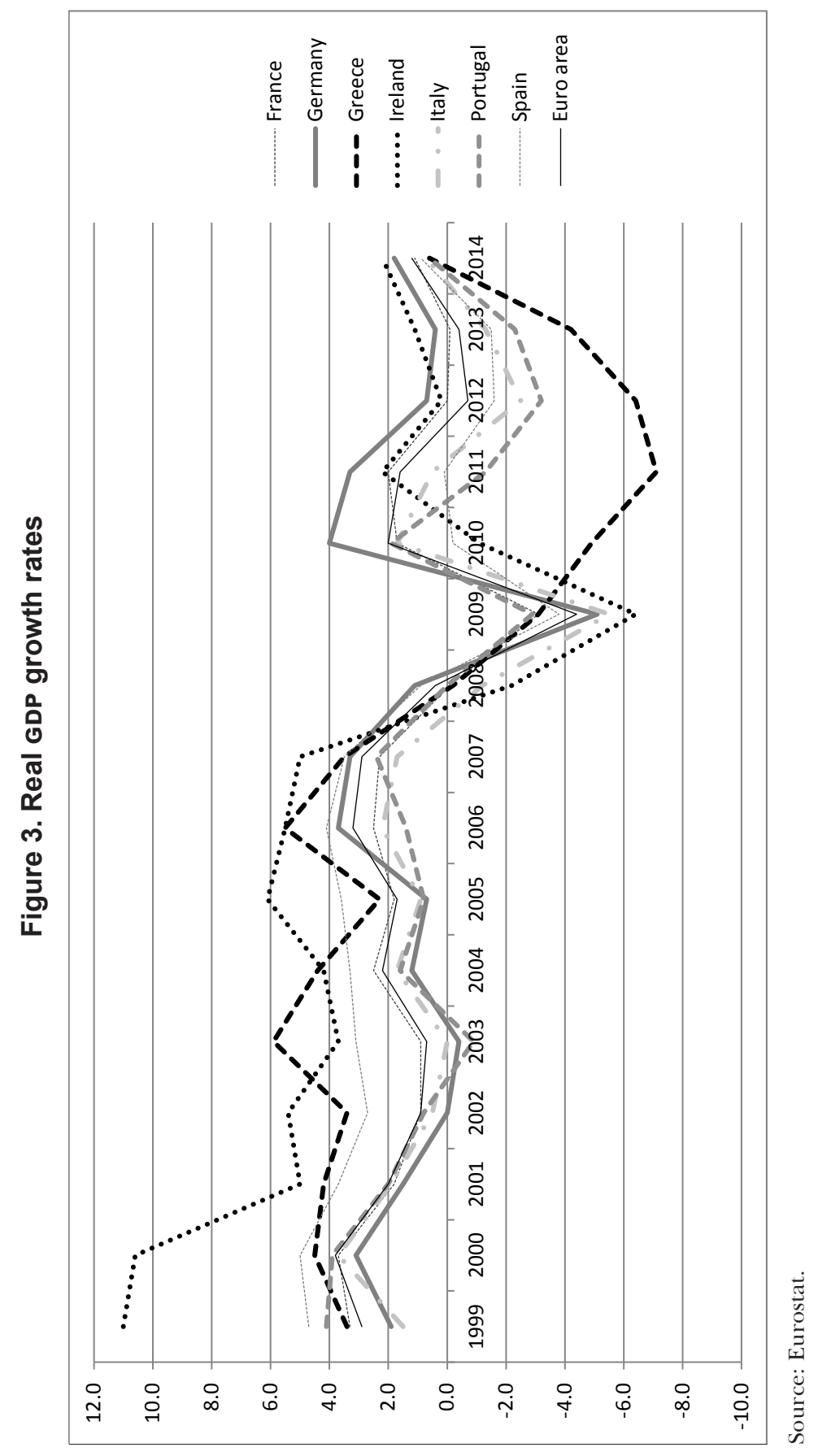


Third, some countries economies are struggling with structural problems. For instance, Portugal was once a textile producing country. Nowadays cheap clothes are produced in Asia and high-quality wear in countries like Italy. Thus, some European economies lack a viable business model. This leads to further problems. Among these problems are labour market distortions which will be discussed in the next section.

\section{The labour market crisis}

The deep recession in Europe had a severe impact on labour markets, too. Increasing unemployment is a typical feature of banking crises (see e.g., Reinhart and Rogoff, 2008). This applies to Europe. Figure 4 shows unemployment rates of selected countries and the average among all Euro members.

According to Brada and Signorelli (2012), differences in labour market performance after recessions can mainly be explained by the quality of institutions, the flexibility of labour markets, and structural factors. We have already seen some economies with structural problems (Portugal). To add another example, at the peak of the real estate bubble in Spain around one in four employees was working in the building sector. The whole sector was under pressure once the bubble burst. A second explanation comes from standard neoclassical theory. Thus, we must look at unit labour costs of some Euro countries (figure 5).

Figure 5 shows unit labour costs on an index basis. Spain's and Portugal's unit labour costs increased over the full time range. Italy's unit labour costs went up until 2009. Germany's unit labour costs, on the other hand, increased until 1996 and then decreased slightly. Ireland is the most impressive case as their unit labour costs went down all the time. But how can the increase in labour costs in Spain and Portugal be explained? Unit labour costs remain constant if nominal wages increase by just the sum of inflation and productivity gains. So why did those countries not achieve productivity gains? Nominal wages increased by much more, forcing unit labour costs up. In addition, labour demand increased as a result of the rise in aggregate demand. However, since capital imports were mainly used for consumption, not investment, productivity only increased modestly. 


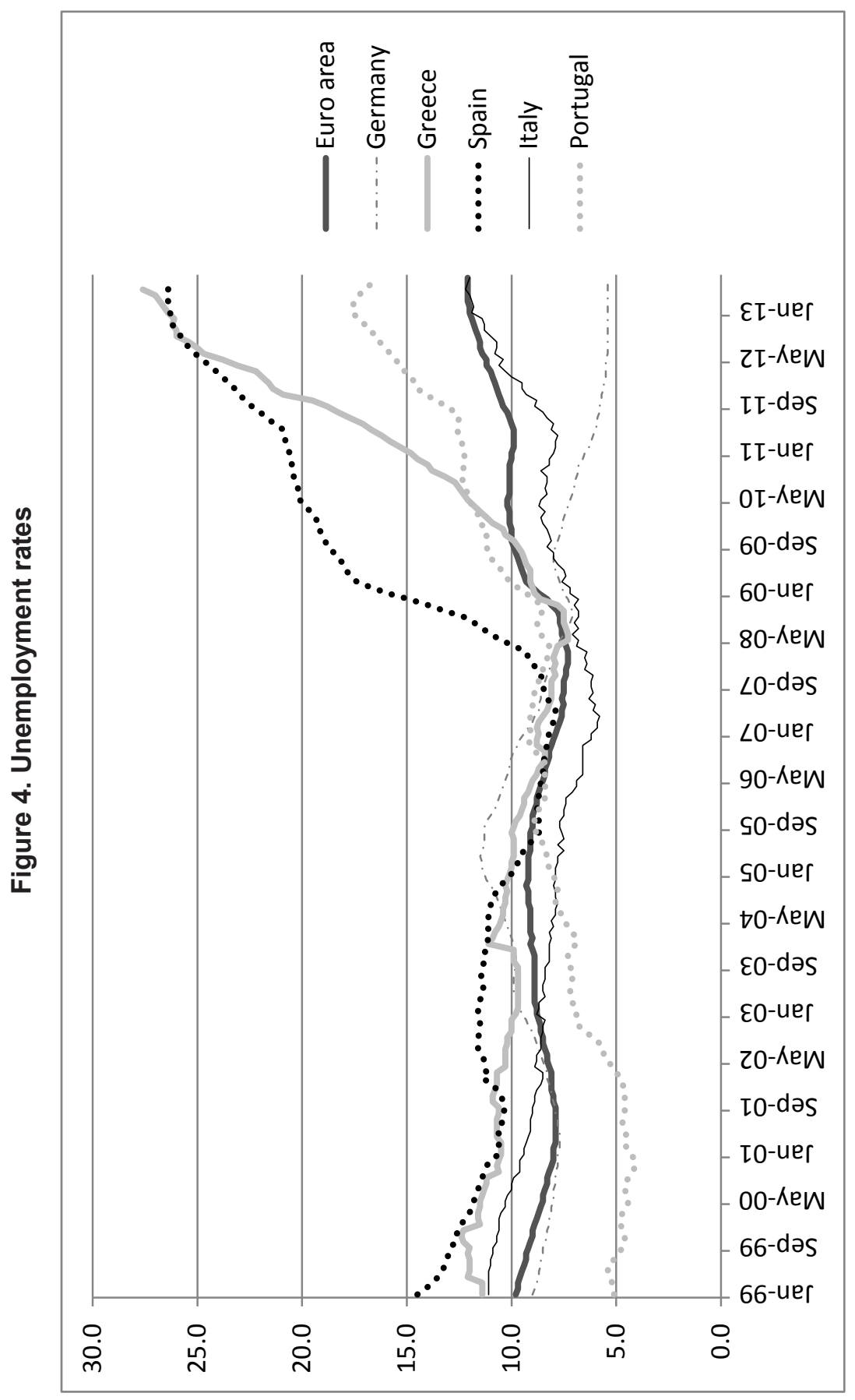

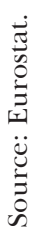




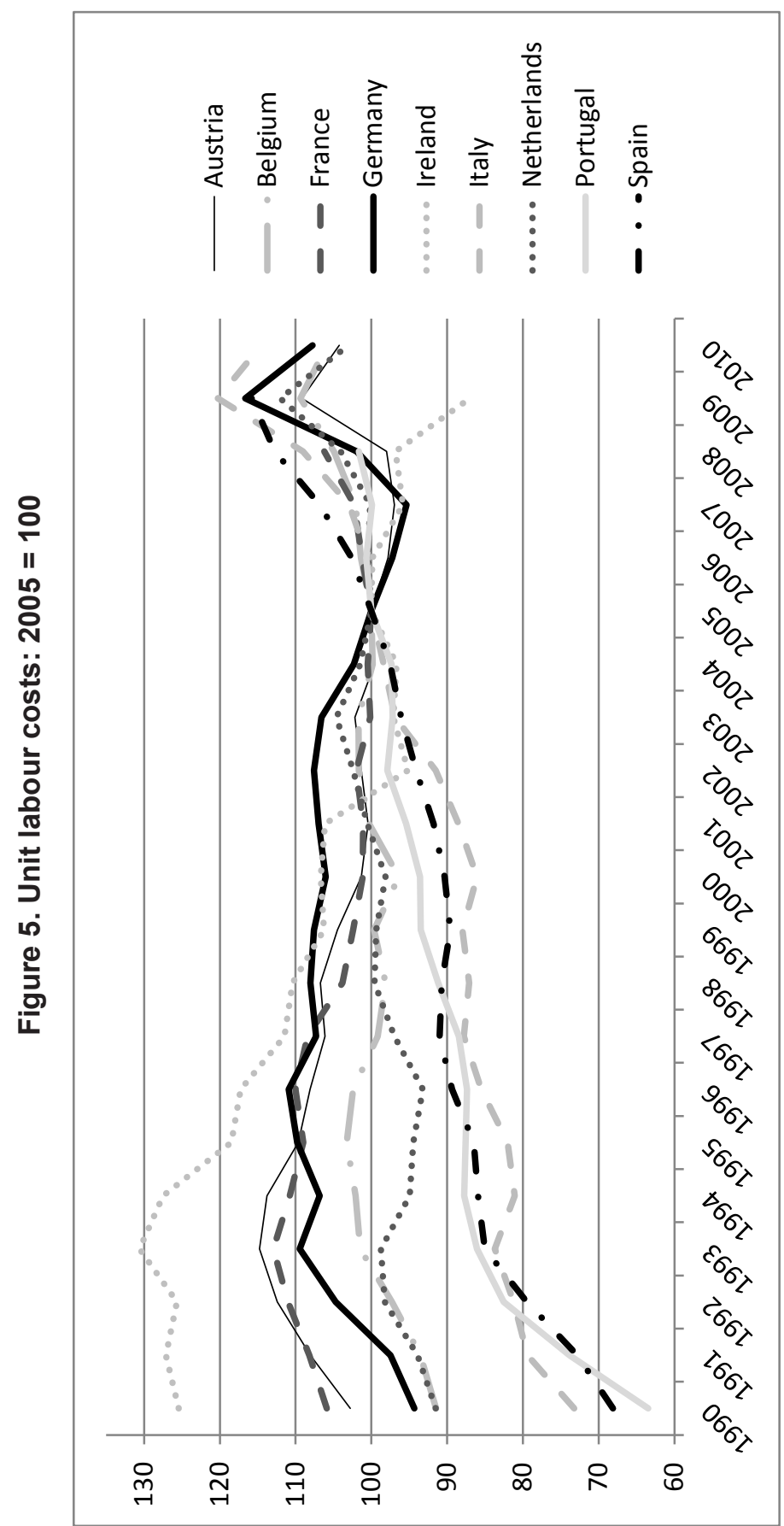

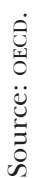


Therefore, these countries lost competitiveness. It should be emphasized that the figure does not tell anything about the absolute differences of unit labour costs between countries. Germany's unit labour costs are still well above those of countries like Greece. However, it is not just costs that matter. One of the competitive advantages of Germany is that it produces high-quality products (like engineering products). In these sectors prices are not the decisive factor; product quality and customer focus count for more (Schröder, 2010). This explains why Germany performs very well even though real wages are relatively high. Wage bargaining might give an explanation for different wage trends.

According to Calmorfs and Driffill (1988), real wages and unemployment are low if wage bargaining takes place either in a decentralized way or centrally. Countries in which wages are negotiated in each sector have, on average, higher unemployment rates due to higher real wages. Trade union density, which might be used as a proxy for centralisation in wage bargaining, is high in Finland while it is slightly above OECD average in Germany, Portugal, and the Netherlands and above average in Spain. Greece, Italy, and Ireland can be considered as countries with trade union density that is neither high nor low. ${ }^{8}$ However, strictness of employment protection might be another factor. According to OECD data, it is among the highest in Portugal. In Germany it is also pretty high. This shows that these data alone cannot explain different labour market developments. However, dismissal protection together with minimum wages are two main factors for high youth unemployment rates in countries like Spain. Lastly, labour market institutions play an important role in explaining unemployment differences. According to the model of Diamond, Mortensen, and Pissarides, there are search frictions on labour markets. Thus, vacancies and unemployment exist at the same. The Beveridge curve (figure 6) illustrates the relation between vacancy $(\mathrm{v})$ and unemployment rates $(\mathrm{u})$. The current status depends on the Beveridge curve and the labour market tension $\Theta$ which is dependent on labour demand and the bargaining power of employees (wage-setting curve).

Simultaneous decreases in the vacancy and unemployment rate require improvement of matching efficiency. Matching efficiency can be

\footnotetext{
${ }^{8}$ Data are taken from Eurostat.
} 


\section{Figure 6. Beveridge curve}
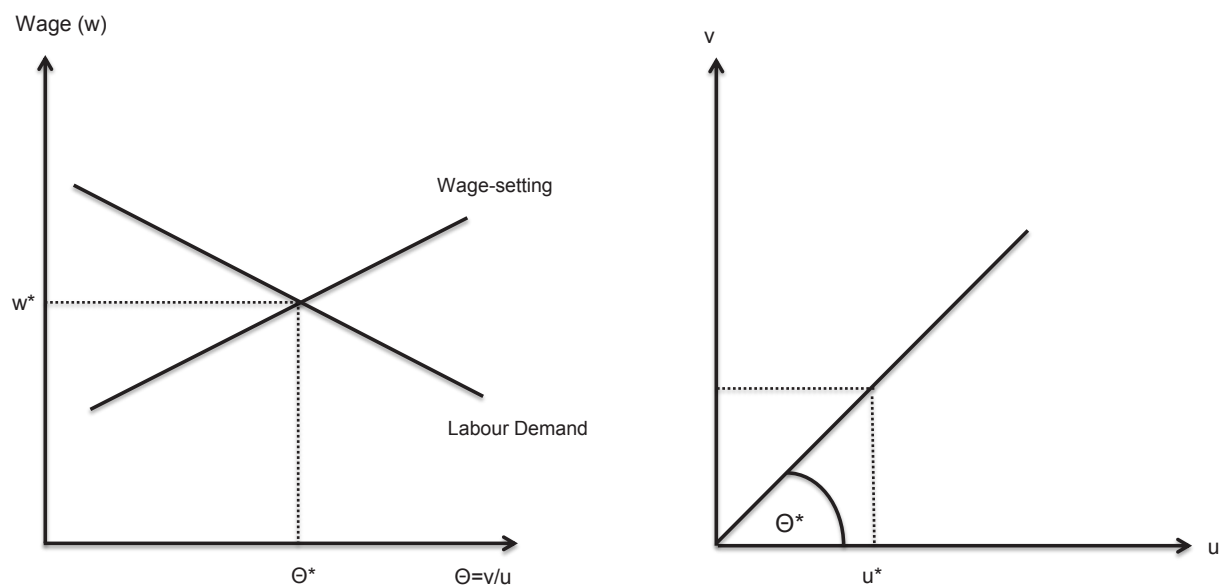

Source: Own illustration.

increased if regional and qualification mismatches fall, i.e., either when the separation rate declines or the matching process improves. Thus, the German labour market "miracle" can be explained by several factors. Germany's national employment agency was reformed in 2003/5. One of the results was that the matching process was improved. The same reform had also intended to force the unemployed to accept low-wage jobs if necessary. Thus, the entitlement period for unemployment benefits was drastically shortened. Labour market flexibility was increased by allowing temporary employment and flexible working hours (in this instance working-time accounts) (Möller, 2010). These reforms taken together caused a remarkable improvement of the situation on the German labour market. Finally, the Beveridge curve was sifted inwards and structural unemployment was reduced. ${ }^{9}$ Thus, the focus of labour market reforms in other

${ }^{9}$ In comparison, Spain is currently in a situation in which the labour market tension $\Theta$ decreased. For instance, the number of employees in the industry, construction, and service sector decreased from 13.1 million to 12.6 million from 2008 to 2012 . At the same time the number of vacancies in these sectors increased from 86000 to 95970 
countries should clearly focus on improving the matching process. Most importantly, regional and qualification mismatches have to be reduced by active labour market policy.

\section{The sovereign debt crisis}

The sovereign debt crisis is sometimes regarded as the main problem in Europe. It is also used as a synonym for the Euro crisis. Some aspects of this crisis have already been covered in section "Causes of the Euro Crisis". In some cases, public debt was already high before countries introduced the Euro, while in others it increased as a result of the financial crisis. On the one hand, large public deficits in the aftermath of the crisis can be explained by rescue measures for banks and companies. On the other hand, public expenditures increased as governments fought the recession (for instance with expansionary fiscal policy) and mandated social expenditures (e.g., unemployment benefits) increased. Moreover, public revenues decreased as a result of lower income and corporate tax payments as well as lower social welfare contributions. This is, however, only one part of the story. For the other, we should look at government bond yields of selected European countries (figure 7).

Note that government bonds yields were different before the Euro was introduced. The nearer the Euro came, the lower the spreads were between German and French government bonds and Italian, Spanish, Portuguese, and Greek government bonds. From 1999 or 2001 on, differences were only marginal. This means that investors trusted Greece just as much as Germany or France. The turning point came in the second half of 2008. From then on, German and French government bond yields fell while they were rising for Greek, Italian, Spanish and Portuguese bonds. Portugal and Greece seem to be the most drastic examples. The spreads between those countries' bond yields and those of Germany changed from $0.13 \%$ (Greece) and $0.00 \%$ (Portugal) in January 2005 to values above $25 \%$ and $10 \%$ within just a couple of years. This does not bolster confidence that investors were entirely rational and took all relevant information into

(Data source: Eurostat). This means that the Beveridge curve shifted outwards, i.e. the matching efficiency worsened. 


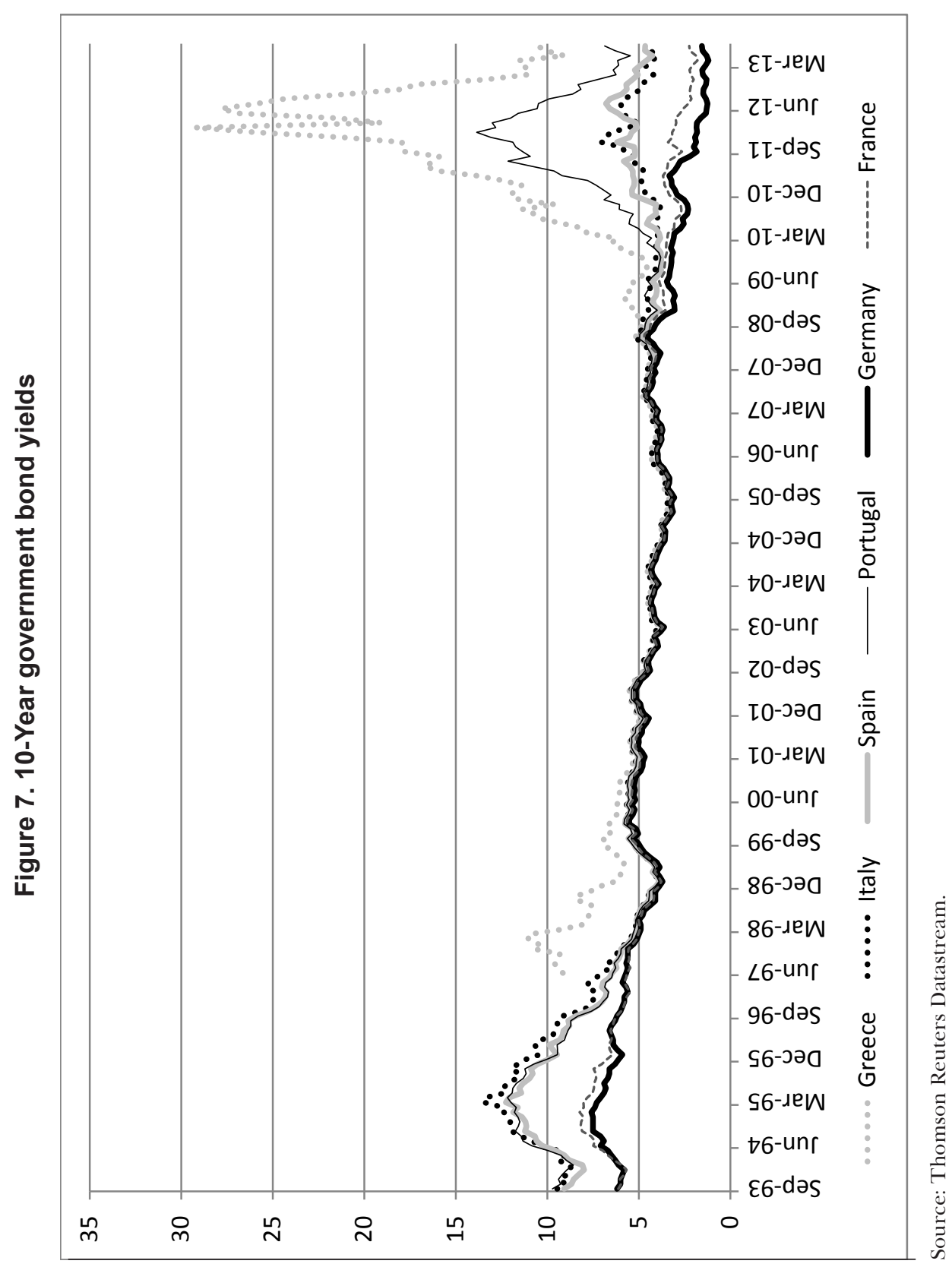


consideration. The same doubts arise if we take a look at ratings. From October 2003 to September 2004, Greek government bonds had a rating of $\mathrm{A}+$ (Fitch), the fifth highest rating that an asset can achieve. Ireland had a rating of AAA until 2009, Portugal AA until September 2009 and Spain $\mathrm{AA}+$ until March 2011. ${ }^{10}$ Greece's rating worsened from A to RD (partial default) within just 2.5 years. This raises the question whether the rating of A was "correct". This is not to blame rating agencies; it is rather to show that most people believed that none of the Euro countries could get in such trouble or that they would be rescued if they did.

Now that public debt has increased due to the financial crisis, countries need to reduce their indebtedness. One problem is private debt (see figure 2). Public and private debt can hardly be reduced at the same time. Countries like the us decided to reduce private debt first. Several European countries conducted austerity measures to reduce public debt. Thus, private debt kept on growing. The latter option seems to be inferior because neither public nor private debt could be substantially decreased. Another issue for reducing debt can be exemplified with the following equation:

$$
\text { (1) } b_{t}=(1+r-g) b_{t-1}+d_{t}
$$

In this equation $b_{t}$ is the debt-to-GDP ratio in year $t, r$ is the interest rate, $g$ is GDP growth and $d_{t}$ is the primary balance in year $t$. The primary balance is the difference between public revenues and expenditures without interest rate payments. The debt-to-GDP ratio can be lowered if GDP growth is higher than the interest rate or if the country achieves a primary surplus $\left(d_{t}>0\right)$. The problem for PIGS countries is that those countries' interest rates are relatively high. We cannot say that they had to pay the interest rates that are illustrated in figure 7 because these are yields and not interest warrants. However, it shows us quite plainly why countries like Greece and Portugal needed help. Let's take the case of Portugal: in 2012 GDP went down by $3.2 \%$ and yields reached a value of around $12 \%$. That means that Portugal would need a primary surplus of more $15 \%$ of GDP just to keep the debt-to-GDP ratio constant. However, Portugal had a primary deficit according to the table 5 .

${ }^{10}$ All data are taken from Fitch Ratings website. 


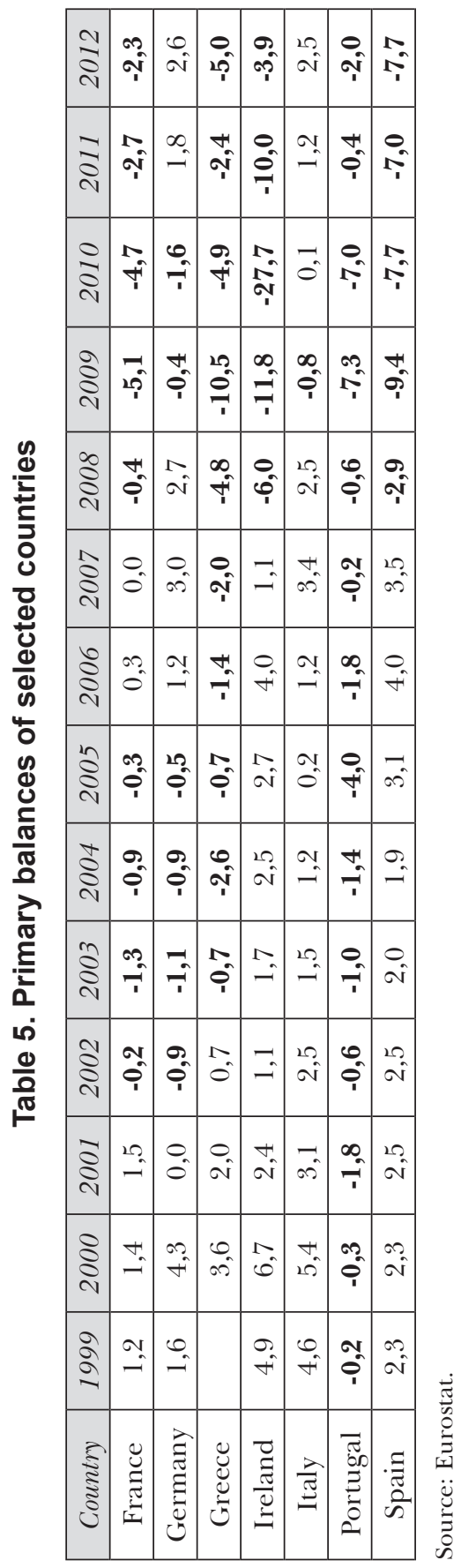


Table 5 shows primary balances of some Euro countries. Bold numbers indicate primary deficits. Portugal had primary deficits in every year. The only reason why the debt-to-GDP ratio did not increase much more before 2008 was that there was some GDP growth up to then. So why did those countries with debt problems not cut spending much more than they did? The problem is simply that they are caught in a dilemma. If a country reduces spending and increases taxes, the primary balance might turn into a surplus. However, these measures also cause GDP to plummet by a higher factor (remember the Keynesian multiplier). In the end, the debt-to-GDP ratio $b_{t}$ might even increase due to this policy. Thus, it is essential for those countries to have GDP growth so that they can grow out of the crisis. GDP growth, however, cannot be the result of increased public spending. It can be caused by expenditure by countries without huge debt problems (e.g., Germany) or by structural reforms. According to the International Monetary Fund ([IMF], 2012), debt reduction is especially successful if there is a policy mix and if monetary policy is loose. Thus, fiscal consolidation should focus on structural problems rather than on temporary austerity measures. As fiscal repression is not likely to be an option for Euro countries, debt has to be reduced cautiously. For instance, primary budget improvements used to be sustainable only if the annual change was around one percentage point. Furthermore, belt tightening can amplify banking problems (Lane, 2012). This all shows that Europe has to accept that it will take a long time to get rid of public debt problems. Austerity measures that focus on short-term debt reductions are not a panacea.

\section{The balance of payment crisis}

Up to now we have only seen problems that are located in single countries. However, Euro countries trade with each other, leading to mutual dependencies. This refers to the problem of increased imbalances in current accounts of Euro member countries. It is instructive to look at the development of current account balances of selected Euro countries (table 6).

Table 6 shows the current account balances of some Euro countries. There are two countries with relatively large current account surpluses, Germany and the Netherlands. Note that Germany ran deficits in 1999 


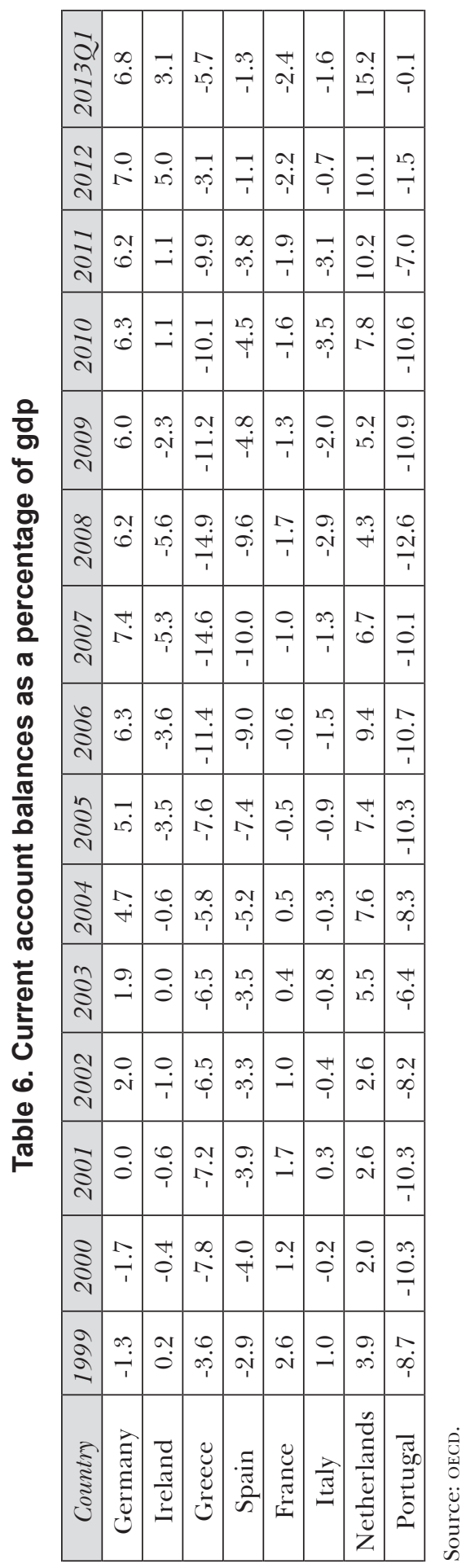


and 2000. Afterwards, the surplus has grown and reached a peak of $7.0 \%$ in 2012. The surplus of the Netherlands is even larger. On the other hand, Portugal, Greece, and Spain run current account deficits. The data are quite worrying as they show massive imbalances in the Euro zone. How can these be explained? Prices and wages increased much more in deficit countries than in surplus countries. Thus, countries like Greece and Spain were faced with real appreciations while Germany "depreciated" against other Euro countries. ${ }^{11}$ At the same time GDP growth rates were higher in deficit countries. As imports depend on domestic income, this provided a further impetus for imports. These deficits and surpluses have further consequences. For euro zone countries the foreign exchange balance is balanced. Thus, current account surpluses are tantamount to capital account deficits and vice versa.

Table 7 shows the financial accounts of selected countries. These are the counterpart of the current account balances. Germany and the Netherlands, which have current account surpluses, have financial accounts deficits. Thus, money is transferred from Germany and the Netherlands to other countries (mainly via credits, portfolio investment, and direct investment). To put it in simple terms: German and Dutch savings are partly used to finance other countries' consumption and investments. The mirror of the financial account deficits of Germany and the Netherlands are financial account surpluses of countries which ran current account deficits. Thus, money flowed from surplus countries to deficit countries like Greece and Portugal. One could also say that these financial account surpluses were necessary to finance those countries' current account deficits. It has to be emphasized that a capital account deficit is synonymous to an increase in external debt if the foreign exchange balance is balanced. Thus, we take a look at the development of external indebtedness which is illustrated in the figure 8 .

Figure 8 shows the international investment positions of selected Euro countries. What is evident from that figure is that Germany's and Netherlands' current account surpluses led to a massive increase in account receivables against other countries. Germany's claims add up to more than

${ }^{11}$ According to Eurostat data, the real effective exchange rate of Germany went down by $8.8 \%$ from 1999 to 2012 . On the other hand, it increased in Spain $(+9.1 \%)$, in Portugal $(+5.1 \%)$, and in Ireland $(+8.5 \%)$. 


\begin{tabular}{|c|c|c|c|c|c|c|c|c|}
\hline $\begin{array}{l}\vec{\partial} \\
\stackrel{m}{a} \\
\vec{v}\end{array}$ & $\begin{array}{l}v \\
0 \\
0\end{array}$ & $\begin{array}{l}\Omega \\
N_{1}\end{array}$ & $\tilde{0}^{\infty}$ & $\stackrel{g}{=}$ & $\begin{array}{l}\tilde{v} \\
\text { ov }\end{array}$ & $\begin{array}{l}0 \\
0\end{array}$ & 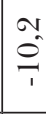 & $\begin{array}{l}0 \\
0 \\
1\end{array}$ \\
\hline$\underset{\sim}{\stackrel{v}{d}}$ & $\begin{array}{l}\infty \\
\infty \\
\infty\end{array}$ & $\stackrel{\infty}{\rightarrow}$ & $\stackrel{\sigma}{-}$ & $\overrightarrow{0}$ & $\dot{0}^{+}$ & $\stackrel{n}{f}$ & की & $\stackrel{F}{F}$ \\
\hline $\bar{ָ}$ & $\begin{array}{l}\mathscr{N} \\
0 \\
0\end{array}$ & $\begin{array}{l}\infty \\
\infty\end{array}$ & $\begin{array}{l}10 \\
\infty\end{array}$ & $\hat{a v}$ & $\stackrel{0}{f}$ & $\stackrel{n}{f}$ & $\begin{array}{l}\infty \\
\infty \\
\infty\end{array}$ & 10 \\
\hline 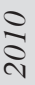 & $\begin{array}{l}0 \\
10 \\
10\end{array}$ & $\begin{array}{l}0 \\
f\end{array}$ & $\stackrel{\sharp}{\sigma}$ & $\vec{F}$ & $\begin{array}{l}0 \\
10\end{array}$ & $\begin{array}{l}20 \\
0\end{array}$ & $\begin{array}{l}10 \\
10 \\
1\end{array}$ & $\begin{array}{l}0 \\
0\end{array}$ \\
\hline$\underset{\overbrace{}}{\stackrel{2}{2}}$ & $\hat{0}$ & $\hat{0}$ & $\begin{array}{l}0 \\
0\end{array}$ & ${ }_{10}^{0}$ & $\begin{array}{l}10 \\
\text { of }\end{array}$ & $\stackrel{0}{0}$ & $\begin{array}{l}\mathcal{N} \\
\end{array}$ & $\stackrel{t}{0}$ \\
\hline $\begin{array}{l}\infty \\
\stackrel{2}{\curvearrowright} \\
\sim\end{array}$ & $\begin{array}{l}0 \\
\stackrel{1}{1}\end{array}$ & $\begin{array}{l}0 \\
\sigma\end{array}$ & $\begin{array}{l}\infty \\
\stackrel{v}{\sim}\end{array}$ & $\begin{array}{l}\tilde{N} \\
\sigma\end{array}$ & ô & $\overrightarrow{0}$ & के & $\exists$ \\
\hline 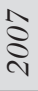 & $\mathbb{N}_{1}^{\infty}$ & $\begin{array}{l}+ \\
0\end{array}$ & $\begin{array}{l}\not{H} \\
\stackrel{v}{\sim}\end{array}$ & $\begin{array}{l}0 \\
0\end{array}$ & $\stackrel{0}{-}$ & $\begin{array}{l}0 \\
\hat{v}\end{array}$ & $\underset{i}{+}$ & $\begin{array}{l}10 \\
\infty\end{array}$ \\
\hline$\underset{\sim}{\stackrel{2}{2}}$ & $\begin{array}{l}0 \\
1\end{array}$ & $\hat{a}$ & $\begin{array}{l}\infty \\
\sigma^{2}\end{array}$ & $\hat{\infty}^{\infty}$ & $\begin{array}{l}\infty \\
\text { of }\end{array}$ & $\begin{array}{l}\infty \\
0\end{array}$ & $\begin{array}{l}0 \\
\dot{1}\end{array}$ & $\begin{array}{l}20 \\
0\end{array}$ \\
\hline$\stackrel{i n}{\stackrel{1}{2}}$ & $\begin{array}{l}\infty \\
20 \\
10\end{array}$ & $\begin{array}{l}\infty \\
? \\
?\end{array}$ & $\begin{array}{l}10 \\
0\end{array}$ & $\hat{0}$ & $\begin{array}{l}\infty \\
\text { of }\end{array}$ & $\stackrel{2}{f}$ & $\begin{array}{l}0 \\
10\end{array}$ & $\vec{\sigma}$ \\
\hline$\underset{\sim}{\stackrel{\nu}{2}}$ & $\begin{array}{l}0 \\
10 \\
1\end{array}$ & $\begin{array}{l}20 \\
0\end{array}$ & $\begin{array}{l}+ \\
\forall\end{array}$ & $\vec{F}$ & $\stackrel{0}{-1}$ & $\begin{array}{l}\infty \\
\infty\end{array}$ & 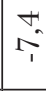 & $\hat{0}$ \\
\hline 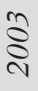 & $\begin{array}{l}0 \\
\text { ov }\end{array}$ & $\underset{1}{0}$ & $\sqrt{20}$ & $\begin{array}{l}\infty \\
\text { of }\end{array}$ & $\stackrel{0}{-}$ & $\stackrel{\infty}{\sim}$ & की & $\stackrel{+}{f}$ \\
\hline 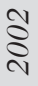 & $\stackrel{\infty}{\rightarrow}$ & $\begin{array}{l}\infty \\
0 \\
0\end{array}$ & $\begin{array}{l}0 \\
0\end{array}$ & $\overrightarrow{a v}$ & $\tilde{0}$ & $\underset{f}{q}$ & $\frac{0}{1}$ & $\hat{0}$ \\
\hline 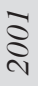 & $\begin{array}{l}0 \\
0 \\
1\end{array}$ & $\begin{array}{l}n \\
0 \\
0\end{array}$ & $\stackrel{r}{*}$ & $\begin{array}{l}\text { ov } \\
\text { of }\end{array}$ & $\hat{\theta}_{1}^{\infty}$ & $\begin{array}{l}t_{0} \\
o\end{array}$ & $\mid \begin{array}{l}\infty \\
0 \\
0\end{array}$ & $\vec{\sigma}$ \\
\hline 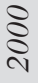 & $\approx$ & $\begin{array}{l}H_{1} \\
\infty\end{array}$ & $\begin{array}{l}10 \\
0 \\
0\end{array}$ & $\overrightarrow{00}$ & $t_{0}^{+}$ & & $\stackrel{\sim}{\sim}$ & $\begin{array}{l}\text { o } \\
\text { s. }\end{array}$ \\
\hline ڤे & $\begin{array}{l}10 \\
0 \\
1\end{array}$ & $\begin{array}{l}\infty \\
\gamma \\
i\end{array}$ & $\begin{array}{c}0 \\
\text { of }\end{array}$ & $\begin{array}{l}0 \\
\text { ov }\end{array}$ & $\begin{array}{l}\infty \\
0 \\
0\end{array}$ & & $\stackrel{n}{7}$ & $\begin{array}{l}0 \\
1\end{array}$ \\
\hline 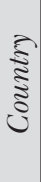 & 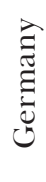 & 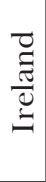 & 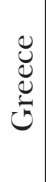 & 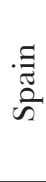 & $\frac{\lambda}{\Xi}$ & 胥 & 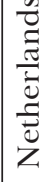 & 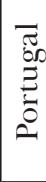 \\
\hline
\end{tabular}




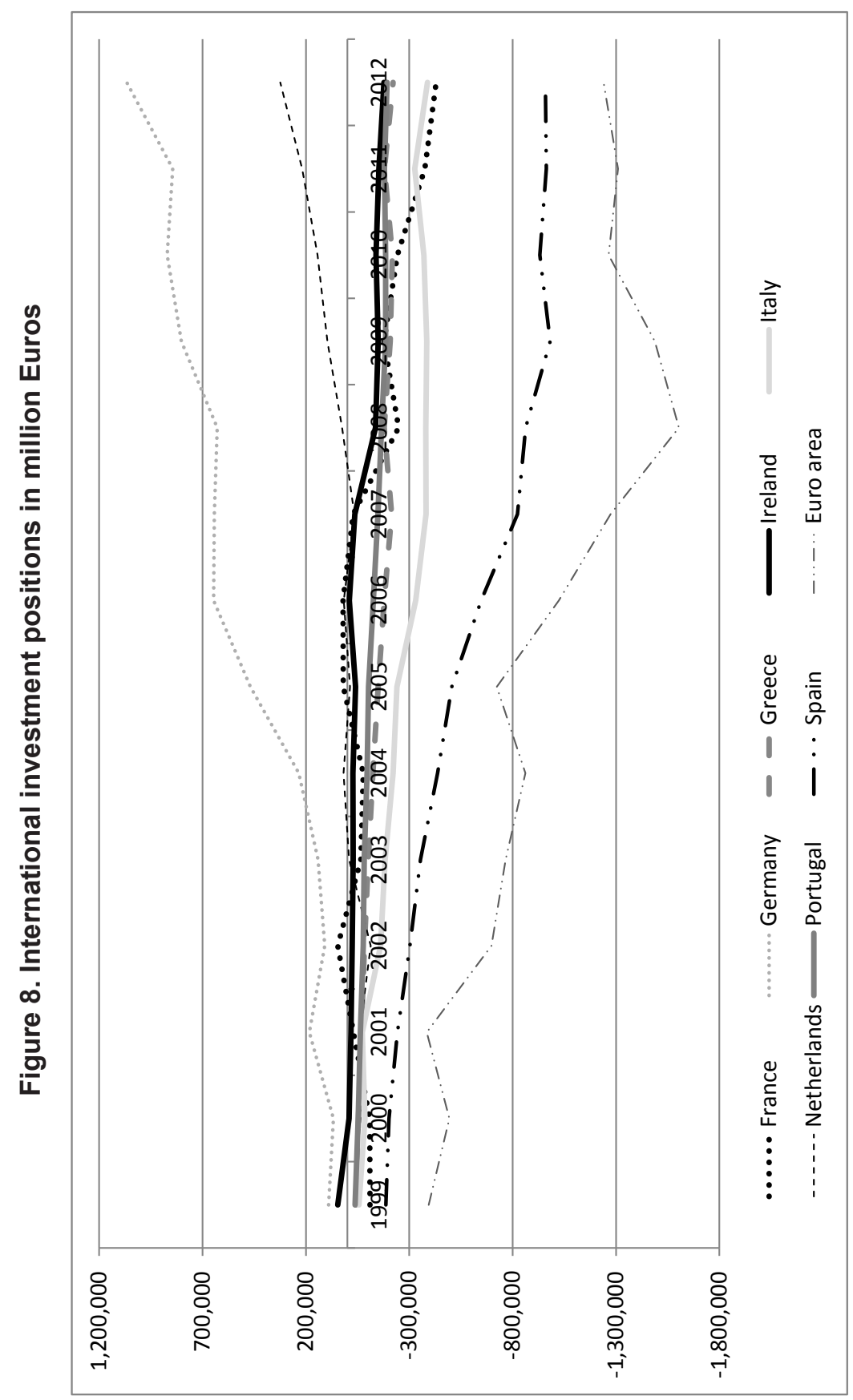

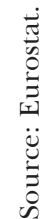


one trillion Euros. These are, of course, not just claims against other Euro countries. At the same time liabilities of countries with current account deficits increased. In the case of Spain the increase is tremendous, around 0.96 trillion Euros. This has far-reaching consequences for most countries. For a country which wants to leave the Euro, Euro-denominated obligations would increase in value (in the new domestic currency). This could trigger sovereign default or at least a massive debt restructuring. If Germany introduced the old D-Mark, its value would doubtless skyrocket. Thus, claims issued in Euro would decline in in D-mark value. This shows why Germany cannot have an interest in a breakdown of the Euro area. Equally, Italy could relatively easily leave the currency without ill effects, since government bonds are mainly hold by domestic savers. Thus, leaving the Euro zone would not be tantamount with an Italian insolvency.

It might seem odd that countries with public debt problems managed to have capital account surpluses even after 2008. We would expect investors to withdraw their portfolio investments because of concern about bankruptcies of banks and companies. If they have serious doubts about the solvency of their debtors, this ought to lead to capital flight. Eventually, this would cause a capital account deficit. According to table 7, large financial account deficits were not a major problem for PIGS countries. To understand why, we have to look at the Euro zone payment system. If a Spanish consumer wants to buy a Roquefort cheese from a French cheesemonger, he tells his house bank to transfer the money from his account to the account of the cheese trader. The Spanish bank transfers the money to the Spanish central bank (Banco de España). Then the Bank of Spain should pass this money on to the European Central Bank (ЕСB). However, this is not how the system works. The Bank of Spain just places an order for crediting this money into the account of the French central bank (Banque de France). Thus, the Bank of Spain now has liabilities vis-à-vis the Есв while the Banque de France has claims against it. The Banque de France then creates new money and credits the amount to the bank of the cheesemonger. Finally, the French bank credits the amount on the cheesemonger's bank account. The result is that money vanished in Spain and new money was issued on France. The same would happen if an Italian saver wanted to invest money in Ireland. Before the crisis liabilities and claims against the Есв balanced out as current account deficits were 
financed by capital imports. However, after the outbreak of the Euro crisis, European banks and investors were not willing to lend as much money to banks and consumers of countries with trade deficits. Moreover, savers and investors withdrew money from troubled countries and invested their money in "safe havens" (e.g., in German government bonds). The effects on the so called Target-balances are presented in the figure 9.

Figure 9 shows the net balance of selected countries with the Euro system. It is remarkable that accounts were more or less balanced until mid-2007. This was the turning point. Afterwards Germany's net claims reached values never before seen. In July 2012 Germany's claims added up to 751 billion euro. Also, the Netherlands' claims increased substantially. On the other hand, the Target balance of countries like Greece, Spain, and Portugal went negative. Italy is a special case as it had a positive balance before 2007 which kept on growing until mid-2009. Then, however, net claims declined and the balance went negative. This cannot be explained by current account deficits but only by capital drain from Italy to other Euro countries. To summarize, these net balances are a mirror picture of current account deficits and surpluses as wells as capital movements. Capital inflows of countries with current account deficits were substituted with Target "credits". This system alone made it possible that countries like Greece could still finance their trade deficits against other countries and that Germany could still have trade surpluses against other Euro member countries. A solution of this capital account crisis can be achieved either by balancing out current accounts or by attracting investors to give money to countries with Target deficits.

\section{Conclusions}

The intention of this article was to demonstrate the causes and the symptoms of the Euro crisis. However, one can draw valuable conclusions from this case study. The conclusions are twofold: first, one could ask whether a currency union is a useful idea at all given that it can lead to severe problems for the member countries. However, there is still overwhelming evidence for the positive effects of currency unions on trade. In their meta-analysis of a magnitude of articles discussing the effect of currency 


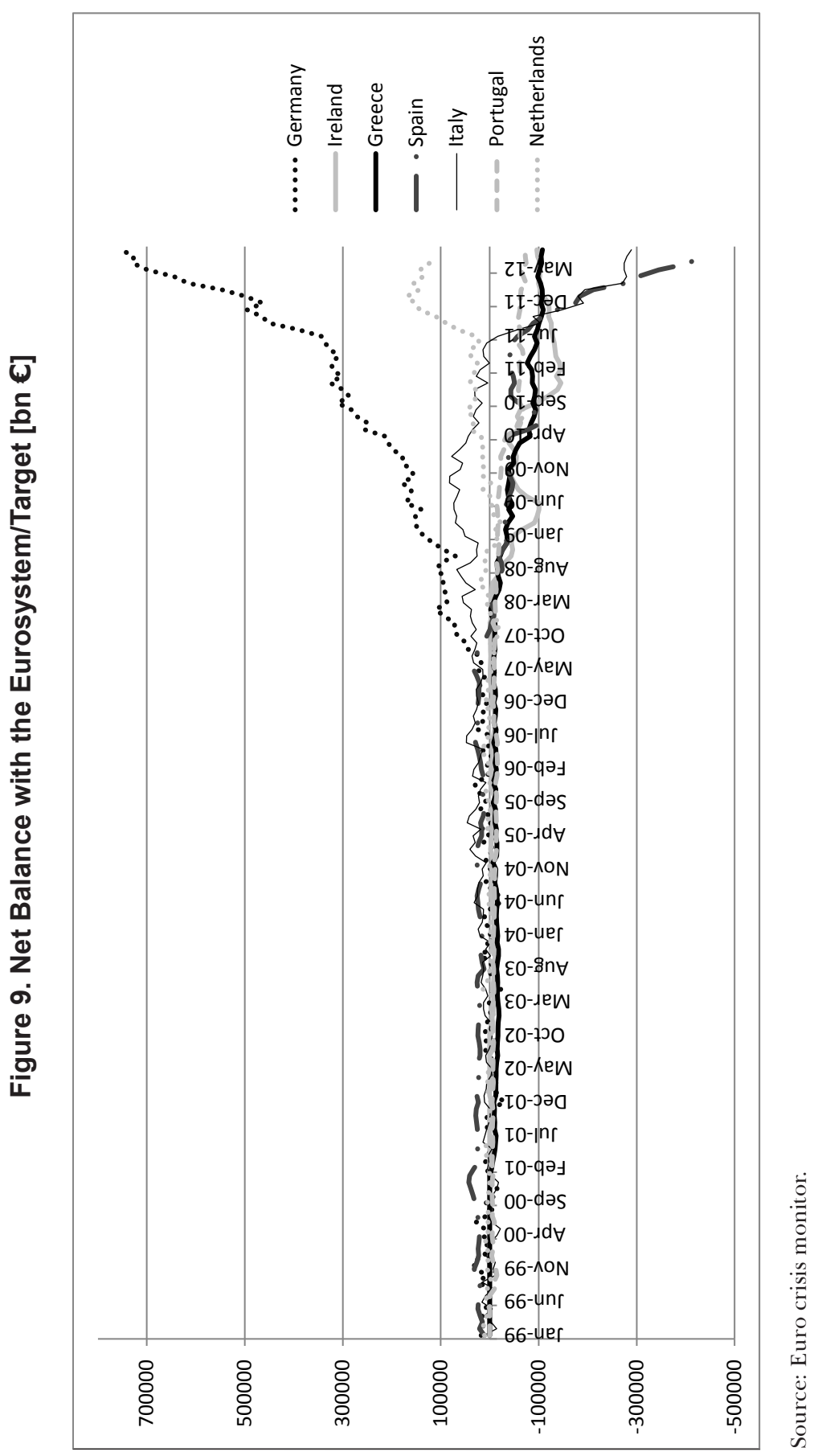


unions on trade, Rose and Stanley (2005) conclude that a common currency increases bilateral trade between two countries by at least $30 \%$. This increase in trade eventually leads to an increase in income of the involved countries (Frankel and Rose, 2002). It is still essential which countries form a currency union or which currency a country adopts as its legal tender. Of course, for Mexico the predicted positive effect of adopting the US dollar is much stronger than joining the Euro area (Frankel and Rose, 2002). The effect should be much stronger if a country uses the currency of one of its major trading partners than otherwise. However, an increase in trade can also be achieved by dollarization (Yeyati, 2003). Thus, there is no need to join or create a monetary union in order to achieve positive trade effects.

Second, the Euro crisis is a warning sign to other countries which are considering the establishment of a currency area. Thus, it is essential to have the Euro zone as an example for do's and don'ts. There are some institutions planning currency unions. Among these are the Association of Southeast Asian Nations (ASEAN) and the Gulf Cooperation Council (GCC). First, each case has to be examined separately. There is some evidence for the usefulness of a monetary union for the ASEAN given that output shocks are highly correlated among member states and that the openness of ASEAN countries is high $(\mathrm{Ng}, 2002)$. There are also good reasons for introducing a currency union among GCC countries (Buiter, 2008). However, one conclusion to draw from the Euro crisis is that a currency union needs effective supranational institutions which monitor central banks and other institutions. Such supranational bodies are missing in the case of the GCC (Buiter, 2008). Furthermore, a currency union has to be prepared for the worst case scenario of a financial crisis (Volz, 2013). This was apparently not the case in the Euro zone. Although the Euro area aimed at reducing the risk of a sovereign default, there was no rescue mechanism that would have helped the Euro zone to cope with financial difficulties of one of its member countries (Buti and Carnot, 2012). The main reason behind that was that the founders of the EMU wanted to prevent moral hazard by high-spending countries. Whilst this is in general a good intention, it led to disorder when countries came into trouble. However, the protections in the Euro area did not suffice. Many countries breached the Maastricht criteria but were not fined. On the other, meeting the criteria was neither 
sufficient to prevent a debt crisis (take Spain and Ireland). The Eu wants to overcome this problem with a scoreboard approach which takes into account a lot of indicators of macroeconomic stability.

A final remark is that financial regulation is a major issue for all countries. Problems in the banking sector in a couple of countries led to a crisis of the whole Euro area. One obvious conclusion is to tighten banking regulation which will be done by adopting the Basel III rules. Furthermore, the Euro area is heading towards a banking union with common banking supervision and a bank resolution mechanism. The idea is that a supranational institution (in this case the ЕСB) supervises the major European banks. In case that a bank is in trouble, the supervisory committee would decide whether to close this bank or not no matter where this bank comes from. In theory, this should reduce the risk of long living zombie banks which live off government subsidies. The current crisis showed that the banking supervision authorities have to act on short notice in order to prevent a long lasting full-blown financial crisis. However, one should always bear in mind that the Great Recession is the most severe crisis after the Great Depression. Thus, the Euro crisis is a phenomenon consisting of many different components. All those individual parts taken together made such a crisis become reality.

\section{References}

Baldwin, R. and Wyplosz, C. (2004). The Economics of European Integration (2 ${ }^{\text {nd }}$ ed.). London: McGraw-Hill.

Brada, J. and Signorelli, M. (2012). Comparing Labor Market Performance: Some Stylized Facts and Key Findings. Comparative Economic Studies, 54(2), 231-250.

Buiter, W. (2008). Economic, Political, and Institutional Prerequisites for Monetary Union among the Members of the Gulf Cooperation Council. Open Economies Review, 19(5), 579-612.

Buti, M. and Carnot, N. (2012). The emu Debt Crisis: Early Lessons and Reforms. JCMS: Journal of Common Market Studies, 50(6), 899-911.

Calmorfs, L. and Driffill, J. (1988). Bargaining Structure, Corporatism, and Macroeconomic Performance. Economic Policy, 3(6), 14-61.

De Grauwe, P. (2012). Economics of Monetary Union. Oxford: Oxford University Press. 
Demary, M. and Schuster, T. (2013). Die Neuordnung der Finanzmärkte: Stand der Finanzmarktregulierung fünf Jahre nach der Lehman-Pleite (IW-Analysen No. 90). Germany: Forschungsberichte aus dem Institut der deutschen Wirtschaft Köln.

Fitch Rating. Retrived August, 2012 from https:/www.fitchratings.com/web_content/ratings/sovereign_ratings_history.xls

Frankel, J. and Rose, A. (2002). An Estimate of the Effect of Common Currencies on Trade and Income. Quarterly Journal of Economics, 117(2), 437-466.

International Monetary Fund (IMF). (2012). World Economic Outlook, October 2012: Coping with High Debt and Sluggish Growth. Washington, Distrit of Columbia: Author.

International Monetary Fund (IMF). (2013). European Union: Publication of Financial Sector Assessment Program (IMF Country Report No. 13/67). Washington, Distrit of Columbia: Author.

Kenen, P. (1969). The Theory of Optimum Currency Areas: An Eclectic View. In R. Mundell and A. Swoboda (Eds.), Monetary Problems in the International Economy. Chicago: University of Chicago Press.

Lane, P. (2012). The European Sovereign Debt Crisis. The Journal of Economic Perspectives, 26(3), 49-67.

McKinnon, R. (1963). Optimum Currency Areas. The American Economic Review, 53(4), 717-725.

Möller, J. (2010). The German Labor Market Response in the World RecessionDe-Mystifying a Miracle. Zeitschrift für Arbeitsmarktforschung, 42(4), 325-336.

Mundell, R. (1961). A Theory of Optimum Currency Areas. American Economic Review, 51(4), 657-665.

Ng, T. (2002). Should the Southeast Asian Countries Form a Currency Union? The Developing Economies, 40(2), 113-134.

Reinhart, C. and Rogoff, K. (2008). Is the 2007 US Sub-prime Financial Crisis so Different? An International Historical Comparison. American Economic Review, 98(2), 339-44.

Rose, A. and Stanley, T. (2005). A Meta-Analysis of the Effect of Common Currencies on International Trade. Journal of Economic Surveys, 19(3), 347-365.

Schoenmaker, D. and Werkhoven, D. (2012). What is the Appropriate Size of the Banking System? (Paper No. 28). Germany: Duisenberg School of Finance Policy.

Schröder, C. (2010). Produktivität und Lohnstückkosten der Industrie im internationalen Vergleich (International Comparison of Productivity and Unit Labor Costs). IW-Trends, 38(4), 1-19.

Volz, U. (2013). Lessons of the European Crisis for Regional Monetary and Financial Integration in East Asia. Asia Europe Journal, 11(4), 355-376.

Yeyati, E. (2003). On the Impact of a Common Currency on Bilateral Trade. Economics Letters, 79(1), 125-129. 